\title{
The Role of Underspecification in Relative Clause Attachment: Speed-Accuracy Tradeoff Evidence
}

February 14, 2022

\author{
Pavel Logačev ${ }^{1}$ \\ ${ }^{1}$ Boğaziçi University \\ Istanbul \\ Turkey
}

\begin{abstract}
A number of studies have found evidence for the so-called ambiguity advantage, that is, faster processing of ambiguous sentences compared to unambiguous counterparts. While a number of proposals regarding the mechanism underlying this phenomenon have been made, the empirical evidence so far is far from unequivocal. It is compatible with several theories, including strategic underspecification (Swets et al., 2008), race models (Van Gompel et al., 2000; Logacev and Vasishth, 2016), and a more recent coactivation-based account (Dillon et al., 2019). While all three classes of theories make matching predictions for the average time to complete $\mathrm{RC}$ attachment in ambiguous compared to unambiguous sentences, their predictions diverge with regard to the minimum completion times. The speed-accuracy tradeoff procedure was used to test the predictions of all three classes of theories. According to a hierarchical Bayesian model, the speed-accuracy tradeoff functions (SATFs) for ambiguous RC attachment conditions show an earlier departure from chance performance than in either high or low attachment conditions. The results further indicate increased asymptotic accuracy in the ambiguous condition but no increase in processing rate. Taken together, this pattern of results is compatible with the strategic underspecification model and coactivation-based accounts, but not with race models.
\end{abstract}

Keywords: keywords

Word count: 11,172 


\section{Introduction}

How does the human sentence comprehension mechanism handle choice points when building structure incrementally? An early and influential idea is the Garden-path model (e.g., Frazier \& Rayner, 1982), which assumes that the sentence comprehension system (hereafter, the parser) is serial, which means that when faced with a structural ambiguity it always makes a choice. The parser is also assumed to be deterministic, which means that given a particular type of structure, the parser will always make the same choice, because its decision-making is based on principles which take into account solely syntactic information and use it to minimize the structure building cost.

Over time, all these claims - seriality, determinism, complete structure building, and the priority of syntax - have come to be challenged. Constraint-based models (e.g., McRae, Spivey-Knowlton, \& Tanenhaus, 1998) abandoned the syntax-first assumption, allowing all kinds of information (syntax, semantics, plausibility, etc.) to be used simultaneously for making parsing decisions. The good-enough processing account (e.g., Christianson, Hollingworth, Halliwell, \& Ferreira, 2001; Ferreira, 2003; Swets, Desmet, Clifton, \& Ferreira, 2008) abandoned the assumption that elaborate structural representations are built no matter what the comprehension task. Later, non-deterministic race-based models such as (Lewis, 2000; Logačev \& Vasishth, 2016; Van Gompel, Pickering, \& Traxler, 2000) abandoned both the syntax-first assumption and determinism, allowing for variability concerning which parse is built from one moment to the next. More recently, the coactivation-based account proposed by Dillon, Andrews, Rotello, and Wagers (2019) has abandoned the idea of competition between simultaneously available parses, counter McRae et al. (1998).

Among all these alternatives to the Garden-path model, the non-determinism proposal of the race-based models is the most provocative because it makes a very surprising prediction that is empirically attested: the ambiguity advantage. Traxler, Pickering, and Clifton (1998) found that among sentences like (1), ambiguous sentences were read faster than their unambiguous counterparts. Specifically, the locus of the ambiguity advantage was at the word moustache, which is the point of disambiguation in (1a) and (1b). (Traxler et al., 1998) found that it was read faster in sentences like (1c), where it did not disambiguate the relative clause attachment, than in sentences like (1a) and (1b), where it did.

a. LOCALlY AMBiguOUS, High ATTACHMENT

The driver of the car [that had the moustache] was pretty cool.

b. LOCALLY AMBiguOUS, LOW ATTACHMENT

The car of the driver [that had the moustache] was pretty cool.

c. GLOBAlly Ambiguous

The son of the driver [that had the moustache] was pretty cool.

I would like to thank Shravan Vasishth for the extensive discussion of many ideas presented here as well as his contributions to this work, Bruno Nicenboim for his help with a variety of issues pertaining to the statistical model used in this paper and for his comments on early versions of this manuscript, as well as two anonymous reviewers whose extensive comments significantly helped to improve the paper.

All data and code associated with this paper are available at https://osf.io/z8tv9.

Correspondence concerning this article should be addressed to Pavel Logačev. Boğaziçi University, Department of Linguistics, Bebek, 34342 Istanbul. E-mail: pavel.logacev@boun.edu.tr 
The aim of this paper is to test the predictions of multiple accounts of the ambiguity advantage using the speed-accuracy tradeoff methodology (e.g., McElree \& Dosher, 1989), with a particular focus on their predictions regarding minimum processing time and asymptotic accuracy in ambiguous compared to unambiguous sentences. The results of the experiment presented here suggest that ambiguous sentences are associated with lower minimum processing times and thus provide evidence against race models.

\section{Accounts of the Ambiguity Advantage}

\section{URM}

To account for the ambiguity advantage effect, Van Gompel et al. (2000) proposed a new model of ambiguity resolution, the Unrestricted Race Model (URM). It assumes that the parser attempts to build all permissible structures simultaneously. As soon as one structure has been constructed, all ongoing structure building is terminated and the structure built first 'wins the race'. Importantly, the time required to construct a particular parse is assumed to be influenced not only by the structural complexity of the parse (as in the Garden-path model), but by all available sources of information, including world knowledge. As a result, the time required to build a particular structure is assumed to vary from sentence to sentence, and because the parser always adopts the interpretation that takes the least time to be constructed, the parser's behavior is non-deterministic.

The explanation of the ambiguity advantage follows from this behavior. In all the sentences in (1), the parser non-deterministically attaches the relative clause either high or low, as soon as it encounters the relativizer that. In some cases, this attachment later turns out to be wrong in (1a) and (1b), and leads to reanalysis when the parser encounters the disambiguating word 'moustache'. No reanalysis is necessary in ambiguous sentences like (1c), because they are compatible with any attachment.

\section{$S M C M$}

Logačev and Vasishth (2016) proposed another race-based model called SMCM (stochastic multiple-channel model of ambiguity resolution) which shares most of the assumptions of the URM, such as that of a race between multiple structure-building processes, as well as the simultaneous availability of all information sources. In contrast to the URM, however, it assumes that RC attachment takes place after the RC has been processed. Therefore, its explanation of the ambiguity advantage in the ambiguous conditions is not based on the absence of reanalysis in the ambiguous conditions, but rather on statistical facilitation (Raab, 1962 as cited in Miller, 1982) in the ambiguous condition: It predicts shorter average $\mathrm{RC}$ attachment times in ambiguous sentences compared to unambiguous sentences due to a smaller proportion of relatively long $\mathrm{RC}$ attachment times compared to their unambiguous counterparts, which is the result of a race between two attachment processes in ambiguous sentences.

Figure 1 illustrates the logic of stochastic facilitation. According to the SMCM, when two attachment processes are engaged in a race, the $\mathrm{RC}$ attachment time in ambiguous conditions is unlikely to be long, since that can only happen if both attachment processes require a lot of time. In unambiguous conditions, on the other hand, only one attachment process determines the $\mathrm{RC}$ attachment duration and long $\mathrm{RC}$ attachment completion times 


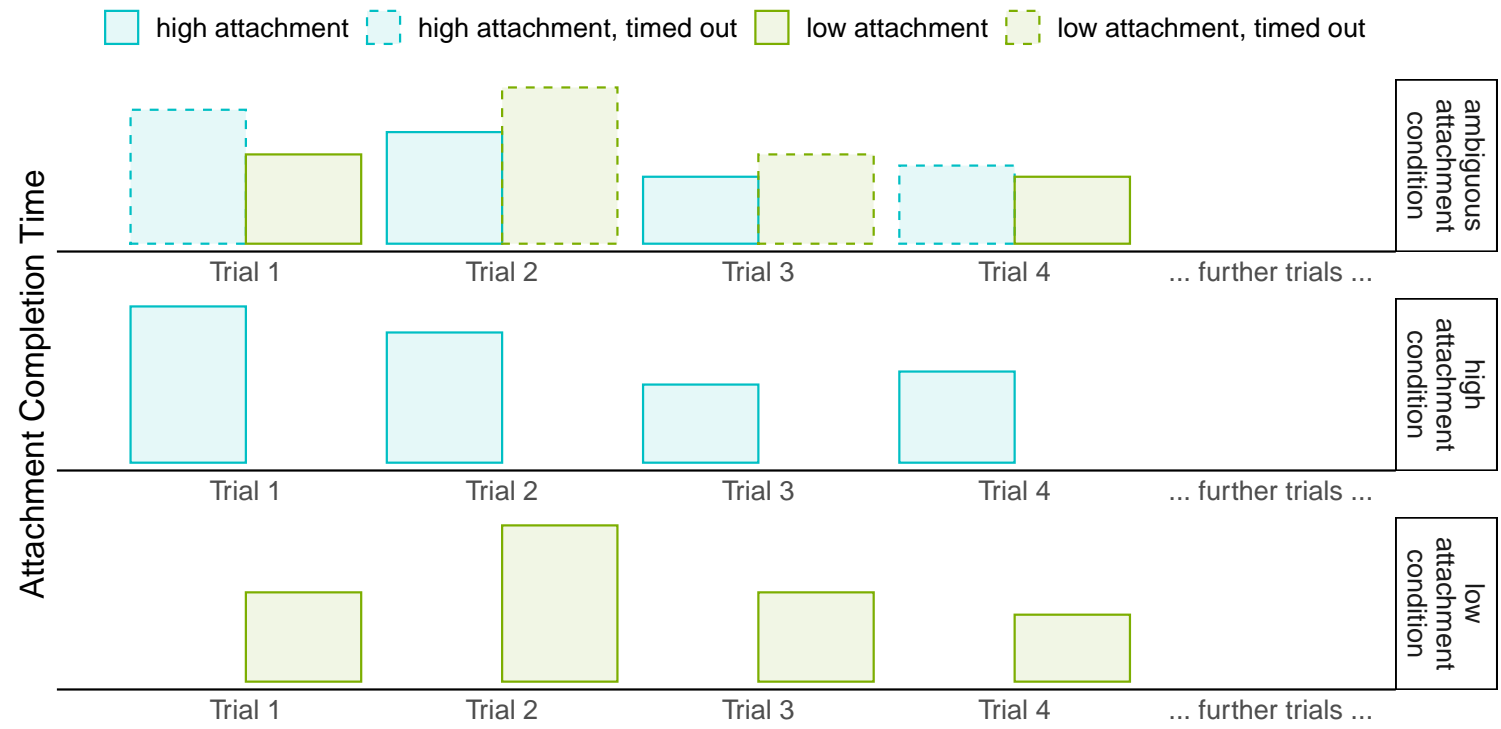

Figure 1

Illustration of the logic of the statistical facilitation predicted by the SMCM. Only a specific attachment process can succeed in unambiguous conditions (middle and lower panel), while the fastest of two attachment processes succeeds in the ambiguous condition (upper panel).

are thus much more likely. The lower probability of long completion times in the ambiguous condition means that the mean RC attachment time in the ambiguous condition should be lower and constitutes the predicted statistical facilitation, which is illustrated in figure 1.

\section{Limited-Capacity Race}

The SMCM and URM both assume that the two attachment processes which are carried out in the ambiguous condition operate at the same speed as in the unambiguous conditions. Because of this assumption, both can be characterized as unlimited capacity systems (e.g., Van Zandt, 2002), as the execution of multiple processes does not decrease the speed of either of the processes compared to when each process is executed alone.

Importantly, there is at least one well-known mechanism that assumes a limited capacity race. In the cue-based parsing framework (Lewis \& Vasishth, 2005), which is based on the ACT-R architecture (Anderson et al., 2004), relative clause attachment can be conceptualized as cue-based memory retrieval of the attachment site. Under this account, the attachment site in sentence like in (1) would be retrieved with retrieval cues based on the meaning of the relative clause, such as [+human]. In the unambiguous sentences $(1 \mathrm{a}, \mathrm{b})$, only one memory trace would match those retrieval cues. In the ambiguous condition (1c), however, there are two matching memory traces, since both son and driver are [+human] and can have a moustache. Therefore, the parser may sometimes retrieve one noun phrase and sometimes the other.

Recently, Nicenboim and Vasishth (2018) have demonstrated that when multiple 
memory traces can be retrieved, the ACT-R retrieval process is equivalent to a race between multiple retrieval processes. In a model of $\mathrm{RC}$ attachment based on cue-based retrieval, the retrieval latency and therefore the attachment time of the $\mathrm{RC}$ are determined in large part by the degree of match between retrieval cues and the memory trace, weighted by the degree to which the cues uniquely identify the memory trace. Importantly, the fact that in the ambiguous condition, not one, but two noun phrases match all retrieval cues results in a cue-overload (Vasishth, Nicenboim, Engelmann, \& Burchert, 2019), i.e., a reduction in the uniqueness of those cues, and thus increases the latencies of the retrieval processes involved in the race. The increase in retrieval latencies due to decreased uniqueness of retrieval cues is also known as the fan effect (Anderson \& Reder, 1999). Importantly, the fan effect imposes an indirect capacity limitation, in that each individual retrieval process is assumed to be slowed when it is executed alongside another retrieval process. Whether the cue-based parsing model predicts an ambiguity advantage depends on the extent to which the expected statistical facilitation due to the race is offset by the fan effect. Importantly, some parameter combinations predict an ambiguity advantage. ${ }^{1}$

\section{Coactivation Models}

An important alternative to limited-capacity and unlimited-capacity models are socalled super-capacity models, in which the speed of a process increases when it runs alongside a competing process (Van Zandt, 2002). Models of this type have been proposed in the context of the redundant signal paradigm (Miller, 1982), in which participants have to respond as quickly as possible to a signal presented on one of two channels (auditory or visual), or on both channels simultaneously (redundant signal condition). Participants respond faster in the redundant signal condition than in either of the single channel conditions. Miller has argued for 'coactivation models' of this phenomenon, 'in which signals presented on different channels contribute to a common pool of activation that initiates a response' rather than 'separate-activation models' which are equivalent to an unlimited capacity race in which response-related activation associated with each signal remains separate from the other channel's activation, and a response is triggered when one of the two activation pools exceeds a certain threshold.

Dillon et al. (2019) have recently argued in favor of coactivation of parses in sentence processing. While they did not propose a specific mechanistic model implementing such coactivation, it could be modeled along the lines of a sequential sampling model such as the Drift Diffusion Model (Ratcliff, 1978; Ratcliff, Smith, Brown, \& McKoon, 2016) in which the sentence processor accumulates evidence for the existence of a valid parse for the current input. Under the assumption that the rate of evidence accumulation depends on the number of parses or their total probability mass (Levy, 2008), evidence would accumulate at a higher rate in ambiguous sentences compared to their unambiguous counterparts, resulting in an ambiguity advantage.

\footnotetext{
${ }^{1}$ This prediction hold regardless of whether $\mathrm{RC}$ attachment is assumed to take place only once the RC has been fully processed (as assumed by the SMCM) or whether it takes place immediately after encountering the relative pronoun (as assumed by the URM).
} 


\section{Underspecification}

Swets et al. (2008) proposed a radically different explanation for this phenomenon, which is grounded in the Good-Enough approach to sentence comprehension (Christianson et al., 2001; Ferreira, 2003). According to their account, the parser underspecifies RC attachment in ambiguous sentences unless task demands require it to commit to a specific reading of the ambiguity. The underlying rationale is that not making any commitment is less costly than committing to one reading and building the corresponding structure. Swets et al. (2008) argued that the task demands in the studies attesting an ambiguity advantage (Traxler et al., 1998; Van Gompel, Pickering, Pearson, \& Liversedge, 2005; Van Gompel et al., 2000; van Gompel, Pickering, \& Traxler, 2001) did not require the disambiguation of $\mathrm{RC}$ attachment, because it was never probed in comprehension questions. In those studies, occasional superficial questions were asked to keep participants focused on the task, but questions about RC attachment were never asked to avoid drawing participants' attention to the attachment ambiguity. Swets et al. argue that under such circumstances, underspecification of $\mathrm{RC}$ attachment can be a feasible strategy for reducing processing effort. In consequence, their underspecification account assumes that the parser avoids any unnecessary commitment and avoids attaching the $\mathrm{RC}$ in ambiguous sentences in order to save time.

Swets et al. tested this proposal in a self-paced reading experiment with sentences like (2), in which different groups of participants were asked different types of comprehension questions about the experimental sentences. They found an ambiguity advantage when comprehension questions were superficial, but did not find such an effect when participants were always asked about the RC attachment after every sentence. They further found an across-the-board slowdown in reading at the relative clause region in the RC questions group, with a larger slowdown in the dispreferred high attachment conditions.

a. LOCALLY AMBiguOUS, High ATTACHMENT

The son of the princess [who scratched himself in public] was terribly humiliated.

b. LOCALLY AMBiguOUS, LOW ATTACHMENT

The son of the princess [who scratched herself in public] was terribly humiliated.

c. GLOBAlLy Ambiguous

The maid of the princess [who scratched herself in public] was terribly humiliated.

While this finding demonstrates that reading is indeed influenced by task demands, it does not entail that parsing depends on task demands, or that readers underspecify. One piece of evidence to that end is a reanalysis of the Swets et al. data by Vasishth (2021), which shows that Swets et al's key finding, an interaction between question type and the effect of RC attachment, is significant only if untransformed reading times are analyzed with an ANOVA. In an ANOVA analysis of log-reading time, or an analysis with linear mixed effects models (untransformed or log-transformed) the interaction fails to reach significance. This indicates that the Swets et al. data does not provide unambiguous evidence for strategic underspecification if both by-participants and by-items variance are accounted for simultaneously as in linear mixed-effects models (Pinheiro \& Bates, 2000). Another piece of evidence against underspecification as the only interpretation of Swets et 


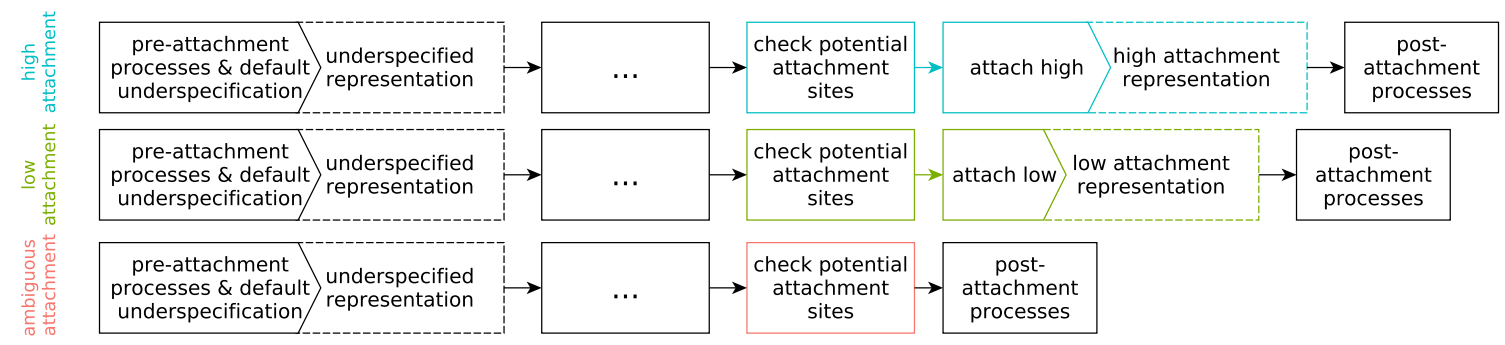

Figure 2

Obligatory Underspecification: The parser's actions in all three attachment conditions when task demands permit underspecification.

al.'s findings is a computational implementation of the SMCM (Logačev \& Vasishth, 2016) which shows that according to the SMCM, a substantial reduction in the magnitude of the ambiguity advantage in the $\mathrm{RC}$ questions condition is expected to the point where it is barely detectable as an artifact of slowed reading in these conditions. Thus, while Swets et al.'s findings are incompatible with the URM under very specific statistical assumptions, they are compatible with both the SMCM and the underspecification account. Importantly, Swets et al.'s proposal allows for multiple mechanisms of underspecification, which will be referred to as obligatory underspecification and selective underspecification in the following.

\section{Obligatory Underspecification}

One interpretation of the underspecification account is that whenever the parser encounters a relative clause, it immediately underspecifies its attachment when the task demands allow it to do so. The parser's actions according to this account are illustrated in figure 2: When it first encounters a relative clause, the parser underspecifies RC attachment in all conditions and proceeds to check whether the ambiguity has been disambiguated at every new incoming word. In unambiguous sentences, it ultimately encounters disambiguating material, and then attaches the RC either high or low. Since no such material is encountered in ambiguous conditions, the $\mathrm{RC}$ is never attached, which amounts to the omission of an entire processing step. If task demands do not permit underspecification, the parser disambiguates ambiguous $\mathrm{RC}$ attachment, either at the end of the $\mathrm{RC}$, or when its core arguments have been processed.

\section{Selective Underspecification}

An alternative interpretation of the underspecification mechanism is illustrated in figure 3. Under this interpretation, the parser delays RC attachment, either until the end of the $\mathrm{RC}$ or at least until its core arguments have been processed. When the required part of the RC has been processed, the parser checks the number of available attachment sites. If only one is available, it builds the appropriate structure (either high or low attachment). If several sites are available, and the task demands permit, the parser creates an underspecified representation. If the task demands do not permit underspecification, the parser disambiguates the $\mathrm{RC}$ attachment at this point. 


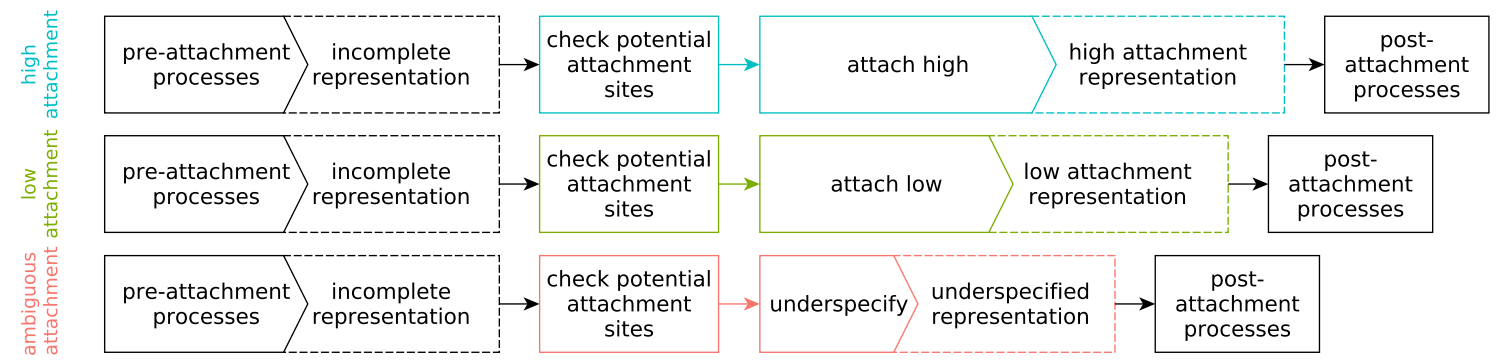

Figure 3

Selective Underspecification: The parser's actions in all three attachment conditions when task demands permit underspecification.

In order to account for the ambiguity advantage, this variant of the underspecification account needs to explicitly stipulate that the underspecification operation requires less time than either high or low attachment. This is in contrast to obligatory underspecification, where such a stipulation is unnecessary as the ambiguity advantage is a direct result of the omission of the attachment step.

\section{Further accounts of the ambiguity advantage}

A number of other accounts of the ambiguity advantage have been proposed, such as surprisal (Hale, 2001; Levy, 2008), as well as in constraint-based models and self-organized sentence processing (Green \& Mitchell, 2006; Smith, 2018; Vosse \& Kempen, 2009). For instance, Levy's independently motivated surprisal account posits that the average processing difficulty of each word is proportional to the word's surprisal value, that is, to its negative conditional log-probability, given the preceding context. Because in ambiguous sentences like (1c), the word moustache is compatible with two sentence structures, but with only one in locally ambiguous sentences like (1a) and (1b), the conditional probability of moustache in ambiguous sentences is higher than in unambiguous sentences. Because higher conditional probability corresponds to lower surprisal, the surprisal account predicts shorter reading times in globally ambiguous conditions compared to the locally ambiguous conditions. Meanwhile, the self-organized parsing account accounts for the ambiguity advantage by positing a processing difficulty in unambiguous sentences like $(1 \mathrm{~b}, \mathrm{c})$ relative to ambiguous sentences like (1a) due to the availability of an infelicitous parse ("the car that had the moustache') in the former, but not in the latter. These models will not be discussed in the following because they make no clear predictions for minimum processing time in ambiguous sentences relative to their unambiguous counterparts, which is the main focus of the present paper.

\section{Distinguishing between race models and underspecification}

Von der Malsburg and Vasishth (2013) present further evidence consistent with Swets et al.'s (2008) underspecification account under certain assumptions. They conducted an eye-tracking experiment in which they compared locally ambiguous and unambiguous Spanish sentences involving adverbial clause attachment. One of their key findings was that 
readers with low working memory capacity read the ambiguous region of locally ambiguous sentences faster than the corresponding region of their unambiguous counterparts. No such difference was observed for high-capacity readers.

These findings are compatible with the underspecification account under the assumption that in addition to being subject to task demands, underspecification tends to be a more common strategy among low-capacity readers than high-capacity readers because underspecified attachment saves memory resources. According to this account, low capacity readers underspecify ambiguous sentences more often than high-capacity readers do, in order to save memory resources, and as a side-effect, also save time, which surfaces as an ambiguity advantage. Von der Malsburg and Vasishth further observed that in locally ambiguous conditions, trials with relatively long first-pass reading times in the ambiguous region were associated with a higher rate of re-reading in the high-attachment conditions than in the low-attachment conditions. This finding is compatible with an underspecification-based account under the assumption that relatively short first-pass reading times at the ambiguous region are indicative of underspecification, while re-reading is indicative of reanalysis. While von der Malsburg and Vasishth's account of their findings is highly plausible, it isn't entirely compelling because the assumptions linking the use of underspecification to working-memory capacity remain to be verified.

More recently, Dillon et al. (2019) found an ambiguity advantage in a speeded acceptability experiment with sentences like (3). Ambiguous sentences like (3c) were judged acceptable more often than their locally ambiguous counterparts in $(3 \mathrm{a}, \mathrm{b})$. According to Dillon and colleagues, both parallel and serial theories of parsing can in principle account for the ambiguity advantage in accuracy. They assume a linking hypothesis based on Signal Detection Theory (Wickens, 2002) according to which sentence acceptability is underlyingly continuous and is converted into discrete acceptability judgments based on its magnitude relative to a certain threshold (e.g., Bader \& Häussler, 2010; Dillon \& Wagers, 2019). Under this assumption, an ambiguity advantage in acceptability judgements follows from Dillon et al.'s additional assumption that parallel parsing theories (presumably including the underspecification account) posit that the acceptability of an ambiguous sentence is the weighted mean of the acceptability scores of all available parses. In consequence, this account predicts higher compound acceptability scores for ambiguous sentences compared to their unambiguous counterparts, which are associated with a lower likelihood of falling below the threshold for a 'grammatical' response for ambiguous sentences. ${ }^{2}$

Dillon et al. discuss that serial models can also explain the ambiguity advantage in acceptability judgments under the assumption that initially misanalyzed grammatical locally ambiguous sentences, at least sometimes, do not trigger reanalysis and are thus misclassified as ungrammatical. Ambiguous sentences, however, never require reanalysis, which is why no misclassification occurs. This leads to a higher percentage of 'acceptable' responses to ambiguous sentences. The class of serial models that make this prediction include race models such as the URM, but not the SMCM.

(3) Armand spotted ...

\section{a. LOCALLY AMBIGUOUS, High ATTACHMENT}

\footnotetext{
${ }^{2}$ Although other combinatorial schemes appear equally possible, many others such as the maximum acceptability score instead of the weighted average would lead to the same prediction.
} 
... the cousin of the painters [who knits].

b. LOCALLY AMBIGUOUS, LOW ATTACHMENT

... the cousins of the painter [who knits].

c. Globally ambiguous

... the cousin of the painter [who knits].

d. UNGRAMMATICAL

... the cousins of the painters [who knits].

Using a signal detection theory approach, Dillon and colleagues tested a key prediction of serial models like the URM, which is that the distribution of underlying continuous acceptability scores in locally ambiguous conditions should be a mixture distribution. One component of this mixture should be the distribution of acceptability scores for acceptable sentences, as on those occasions, the parser chose the correct structure at the outset, or reanalyzed after an initial misparse. The second component should be the distribution of acceptability scores for unacceptable sentences, as on those occasions the parser chose the structure that later turns out to be incorrect.

Dillon et al. argue that the variance of the presumably bimodal mixture distribution predicted by serial models for locally ambiguous sentences should be substantially larger than the variance in the ambiguous condition, and that this difference in variance should show up as a difference in the shape of the receiver operating characteristic functions (ROC; Wickens, 2002) between experimental conditions. They find no evidence for a larger variance of the underlying continuous acceptability values in locally ambiguous conditions. They further assessed the quantitative fit of both the parallel model and the URM-like serial model to their speeded acceptability data, and found that the parallel model substantially outperformed the URM-like model.

Dillon et al.'s findings provide tentative evidence against URM-like serial models and in favor of parallel representation of ambiguous structures, such proposed by surprisal and possibly by the underspecification account, but it is also compatible with some serial models, such as the SMCM: While the URM predicts that the underlying acceptability scores follow a simple mixture distribution, the SMCM makes a different prediction. Under the assumption that that more acceptable structures are also faster to construct (e.g., Rayner, Warren, Juhasz, \& Liversedge, 2004; Van Gompel et al., 2000) the SMCM predicts that the acceptability score of an ambiguous sentence should be the maximum of the acceptability scores of its two possible structures, rather than a weighted average. It is currently not clear how a computational implementation of this model would fare on Dillon et al.'s data compared to the two models they considered, and if their dataset has the potential to clearly adjudicate between these models. However, it appears that their findings are in principle compatible with some serial models, such as the SMCM.

Importantly, the finding of an ambiguity advantage in a speeded acceptability judgment task appears to be at odds with the obligatory underspecification account (fig. 2). This is because obligatory underspecification assumes that underspecified representations become available at the same point in time in all conditions. As informed acceptability judgments in ambiguous conditions must be based on underspecified representations according to this model, this should also be possible in unambiguous conditions. Because participants were not asked any comprehension questions in Dillon et al.'s experiment, the optimal strat- 
egy would be not to perform any attachment in unambiguous conditions. Assuming that underspecification is indeed strategic, as argued by Swets et al. (2008), the parser should arguably be able to avoid carrying out $\mathrm{RC}$ attachment in unambiguous conditions as it does in ambiguous conditions. As a result, reaction times and accuracy for all attachment conditions should be equal, which is not the case in the Dillon et al. experiment. Thus, Dillon et al.'s finding provides evidence against the obligatory underspecification account.

This logic does not apply to the selective underspecification model (fig. 3). Because an underspecified representation is not created in all conditions, and because it is produced by a specialized operation which is presumably only available under the right kind of task demands, and only if the sentence has been deemed ambiguous, the processing effort in unambiguous conditions cannot be reduced by simply underspecifying unambiguous conditions as well. There are multiple ways in which the selective underspecification can account for the ambiguity advantage in response accuracy in Dillon et al.'s data. One possibility is that because participants were required to respond within 2 seconds, they determined in advance after how much time to respond on any given trial. If the sentence had not been processed before the self-set deadline, participants pressed a random button. Because ambiguous sentences were processed faster, it was more likely that they would be processed before the deadline than unambiguous sentences. A second way to explain this effect is through the additional stipulation that the underspecification process is not only shorter but also less susceptible to failure than either of the attachment processes.

The magnitude of the ambiguity advantage in acceptability judgements is of particular importance in distinguishing between underspecification- and race-based models of ambiguity resolution. Dillon et al. found that sentences in their experiment were judged as acceptable $74 \%$ of the time in ambiguous attachment conditions, but only $60 \%$ and $41 \%$ of the time in unambiguous low- and high-attachment conditions respectively. The SMCM can explain this effect under the assumption that each attachment process (high and low) has a particular probability of being successfully carried out $\left(P_{H}\right.$ and $P_{L}$, respectively), and that a failure to successfully attach the $\mathrm{RC}$ results in an incorrect ungrammatical response in the judgment task. Under the assumption that the two attachment processes are independent, the success probability in the ambiguous condition should correspond to the probability that at least one attachment process is successfully carried out, i.e., $P_{H}+P_{L}-P_{H} \cdot P_{L}$. Using the observed accuracy rates of $60 \%$ and $41 \%$ as estimates of the success probability of the low- and high-attachment processes, respectively, the expected accuracy rate in the ambiguous conditions is $0.6+0.41-0.6 \cdot 0.41=0.764$, i.e., $76.4 \%$, which is quite close to the observed accuracy rate of $74 \%$. Both mechanisms predict a higher accuracy rate in the ambiguous condition, but underspecification makes no quantitative predictions without further assumptions. Thus, while this finding is compatible with the underspecification account, it is expected under the SMCM.

Importantly, all findings based on average RTs are compatible with multiple accounts, which complicates the identification of the mechanism underlying the ambiguity advantage. However, several accounts differ in their predictions regarding the relative shapes of the completion time distributions of attachment in ambiguous and unambiguous conditions. In the following, an experiment using the speed-accuracy tradeoff paradigm (Dosher, 1976) will be presented, which tests the fine-grained predictions of the above-mentioned models regarding timing and accuracy. 
To anticipate the key finding: The results of the experiment show that ambiguous sentences are associated with shorter minimum processing times than either of the unambiguous conditions, which is compatible with the co-activation and the selective underspecification account, but not compatible with any of the race models discussed above.

\section{Speed-Accuracy Tradeoff Methodology}

It has been known since at least Pachella (1974), that many cognitive tasks can be performed more accurately at the cost of lower speed, or faster at the expense of accuracy. Figure 4 illustrates a typical speed-accuracy tradeoff function (SATF) for simple cognitive tasks which describes this tradeoff. During an initial period, participants' performance is at chance level as this interval corresponds to the minimum amount of time required to process the stimulus to the required degree to perform the task. As more time is spent on the task, the likelihood that the relevant cognitive process is completed increases, and as a result so does accuracy, albeit typically with a negative acceleration, approaching the participant's asymptotic accuracy on that task (Dosher, 1976).

In sentence processing experiments, SATFs for specific processes involved in the processing of a particular type of structure can be obtained using the response-signal paradigm. In it, participants are asked to judge the acceptability of a sentence after different amounts of time (e.g., McElree, 1993). In computing the SATF, participants' performance at every time lag is quantified by the Signal Detection Theory sensitivity measure $d^{\prime}$ (Liu \& Smith, 2009; Wickens, 2002), which, unlike the percentage of accurate responses, is unaffected by participants' response bias towards either response. To the extent that distinguishing between acceptable and unacceptable sentences requires a particular cognitive process, such as the retrieval of a particular element from memory, or resolving a syntactic dependency between a head and a dependent, the SATF will reflect the operation of that process. Slowed processing in one condition compared to another will be reflected either in a difference between intercepts, or in a difference between rates of the two SATFs, as illustrated in figure 9 (middle and right panel). Meanwhile, differences in the probability of successfully carrying out processes required to perform the main task will surface as a difference in asymptotic accuracy, as illustrated in figure 9 (left panel).

Typical speed-accuracy tradeoff functions in such tasks can be characterized with three parameters which have theoretically interesting interpretations and can provide significantly more information than response times (RT) and accuracies (e.g., McElree \& Dosher, 1989; Wickelgren, 1977). The negatively accelerated shifted exponential function in figure 4 follows equation 1 (Dosher, 1976), and can be characterized by the parameters intercept $\delta$, asymptote $\lambda$, and the rate parameter $\beta$. The intercept $\delta$ corresponds to the point in time when participants' performance departs from chance, and thus corresponds to the minimum amount of time required to process the stimulus to a degree required to carry out the task. The asymptote parameter $\lambda$ corresponds to the maximally attainable accuracy on the task, while the rate parameter $\beta$ describes how quickly the function approaches its asymptote. Its reciprocal, $\beta^{-1}$, coincides with the time the SATF takes to reach $63 \%$ of the asymptote.

$$
d^{\prime}(t)=\lambda \cdot\left(1-e^{-\beta \cdot(t-\delta)}\right), \text { for } t \geq \delta ; 0 \text { otherwise }
$$




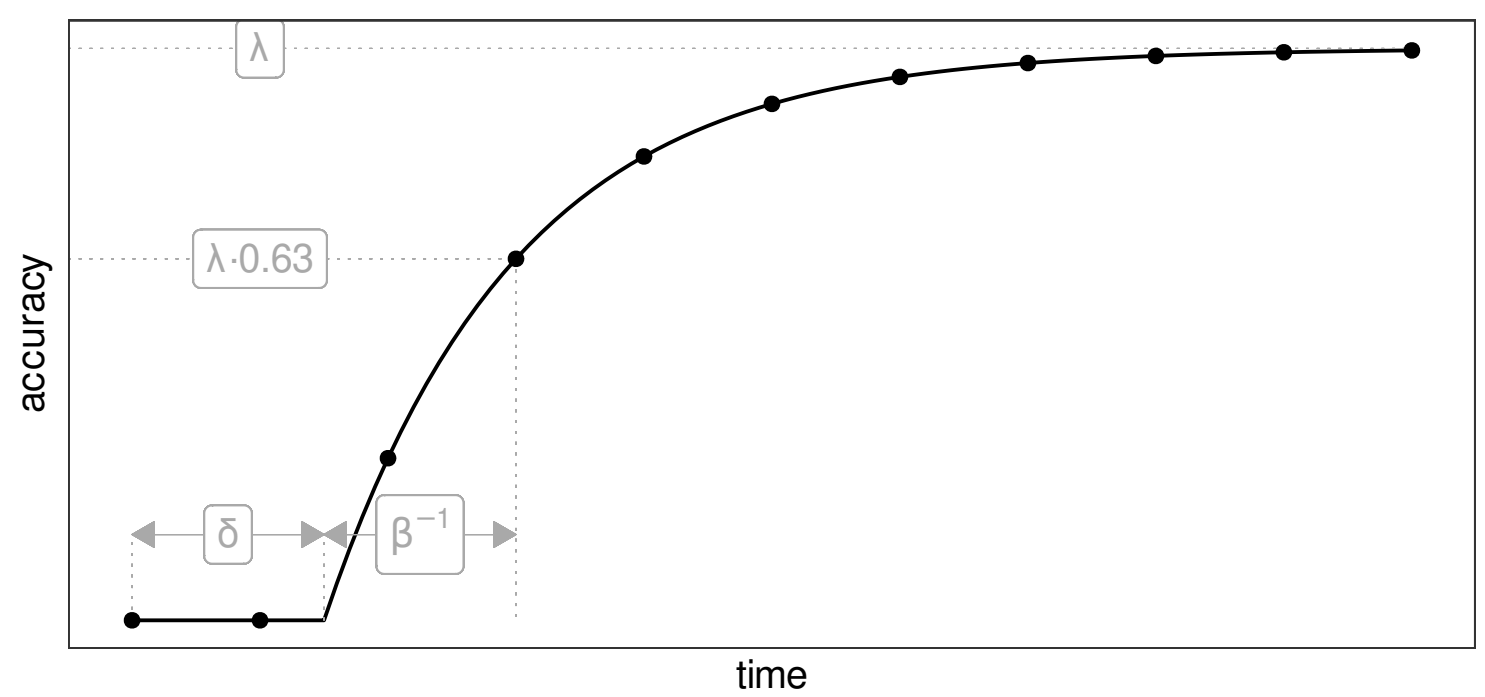

Figure 4

A typical speed-accuracy tradeoff function which can be described by the negatively accelerated shifted exponential function in equation 1. After an initial period of chance performance, accuracy begins to increase at the intercept $(\delta)$. The growth rate $\beta$ determines how quickly function approaches asymptotic performance $(\lambda)$. The reciprocal of the rate $\beta^{-1}$ can be interpreted as the time required for the function to reach approximately $63 \%$ of the asymptote.

\section{condition - a $\cdots \cdot$ b}
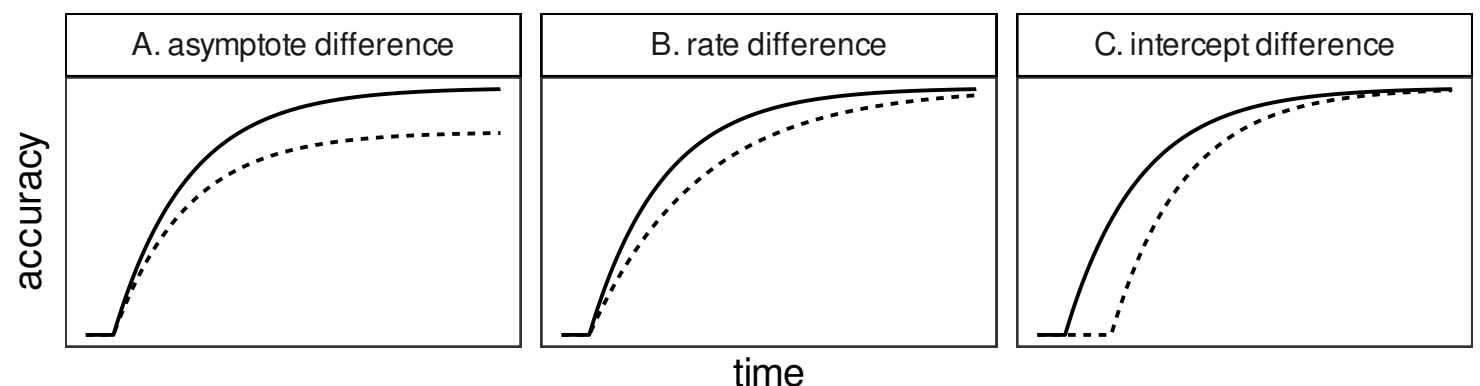

Figure 5

Hypothetical differences in each of the three parameters (asymptote, rate and intercept) of the speed-accuracy tradeoff functions in two conditions. 


\section{SAT and Relative Clause Attachment}

In order to test fine-grained timing predictions of the underspecification account, the coactivation account, and both types of race models regarding timing and asymptotic accuracy, I used the response-signal paradigm (Liu \& Smith, 2009; McElree, 1993; Wickelgren, 1977) to compare the time-course of processing relative clause attachment in German sentences like (4) with ambiguous and unambiguous RC attachment. In contrast to their English equivalents in (1), (2), and (3), relative clause attachment in the unambiguous conditions in (4) is disambiguated at the first word of the RC by means of gender match between the relative pronoun and the available attachment site(s). Because singular noun phrases including (relative) pronouns in German carry gender-marking, the attachment of a relative clause is fully determined by the gender of its relative pronoun when all possible attachment sites are singular.

For example, in the high-attachment sentence (4a), the relative clause with the relative pronoun 'der' must modify the noun phrases headed by a masculine noun ('Manager'; manager-masc), whereas in the low-attachment sentence (4b), it must attach to 'Sänger' (singer-masc) because the alternative ('Managerin') is feminine, and therefore cannot be referred to by a masculine relative pronoun. In sentence (4c), either attachment is possible. The sentence in $(4 \mathrm{~d})$ is unacceptable because no attachment is possible as the gender of the relative pronoun does not match the gender of any of the structurally available NPs. This experimental design ensured that the acceptability of a sentence could not be established without attaching the relative clause. While the models make the same predictions for simple reading times and reaction times, their predictions for speed-accuracy tradeoff profiles of the sentences in (4) diverge. The predictions of each class of theories will be discussed in turn.

a. HIGH ATTACHMENT

Was dachte der Manager der Sängerin, [der schwieg]? What thought the manager.MASC (of) the singer.FEM, who.MASC was silent 'What did the manager of the singer who was silent think?'

b. LOW ATTACHMENT

Was dachte die Managerin der Sängers, [der schwieg]?

What thought the manager-FEM (of) the singer.MASC, who.MASC was silent

c. Ambiguous ATtaCHMENT

Was dachte der Manager des Sängers, [der schwieg]? What thought the manager.MASC (of) the singer.MASC, who.MASC was silent

d. UNGRAMMATICAL

* Was dachte die Managerin der Sängerin, [der schwieg]? What thought the manager-FEM (of) the singer-FEM, who.MASC was silent

\section{Underspecification}

The selective underspecification model in figure 3 assumes that under the right task demands, the parser unambiguously attaches RCs when their attachment is unambiguous, 
and avoids doing so when it is ambiguous. An acceptability judgment task arguably constitutes such a situation, since what is at issue is whether the sentence has a syntactically licensed structure, and not which structure is correct.

Because this account posits that underspecification is carried out faster than either of the $\mathrm{RC}$ attachment processes, it follows that the underspecification process in fig. 3 must have (i) a lower minimum completion time than either of the RC attachment processes, or (ii) a higher processing rate, or (iii) both. In terms of speed-accuracy tradeoff function parameters, this means that the ambiguous condition should have (i) a lower intercept $\delta$ than the unambiguous condition (as in fig. 5C), or a higher processing rate (as in fig. 5B), or both.

While selective underspecification predicts a higher response accuracy in the ambiguous condition in Dillon et al.'s experiment due to the fact that participants had to respond within a limited amount of time, it does not predict a difference in response accuracy if the process is given sufficient time to unfold. Thus, it does not predict any differences in asymptotic accuracy without further assumptions. However, it is compatible with the prediction of higher asymptotic accuracy in the ambiguous condition under the stipulation that shorter processes have a higher probability of being executed successfully.

The predictions of the obligatory underspecification for the German sentences in (4) are less clear because in contrast to sentences like (1b,c), the unambiguous sentences $(4 \mathrm{a}, \mathrm{b})$ are disambiguated at the first word of the relative clause. While the parser can underspecify ambiguous sentences, it cannot do so with unambiguous sentences under the assumptions of obligatory underspecification because they are disambiguated immediately by means of relative pronoun morphology. Because obligatory underspecification makes no assumptions about the relative durations of underspecification and full attachment, but only about their order, it is in principle compatible with any result.

\section{Unlimited-Capacity Race}

Although the SMCM and URM offer different explanations for the ambiguity advantage effects found in English sentences like (1), both assume that whenever an ambiguity arises, the permissible structures engage in a race. Because the unambiguous sentences like (1) are not disambiguated immediately, the URM posits this process at the first word of the ambiguity, while the SMCM assumes that it takes place at a later point during the processing of the RC. Because the unambiguous sentences in (4) are disambiguated at the first word, both models predict that ambiguous conditions should be processed faster due to statistical facilitation. While only high attachment can be performed in high attachment sentences, and only low attachment in low attachment sentences, both structures are built simultaneously in ambiguous sentences. Since the parser adopts the structure which is computed the fastest, relatively fast $\mathrm{RC}$ attachment will occur more frequently in ambiguous sentences than in their unambiguous counterparts. More specifically, the probability of having successfully processed the $\mathrm{RC}$ attachment in the ambiguous condition $\left(F_{A}\right)$ at any given time $t$ equals the probability that either the low attachment or the high attachment process has been successfully completed $\left(F_{H}\right.$ and $F_{L}$, respectively), as in equation 2 .

Figure 6 exemplifies the resulting predictions for the distribution of RC attachment completion times in the ambiguous condition under the assumption that both RC attachment processes are approximately equally fast. It illustrates the predicted relationship 


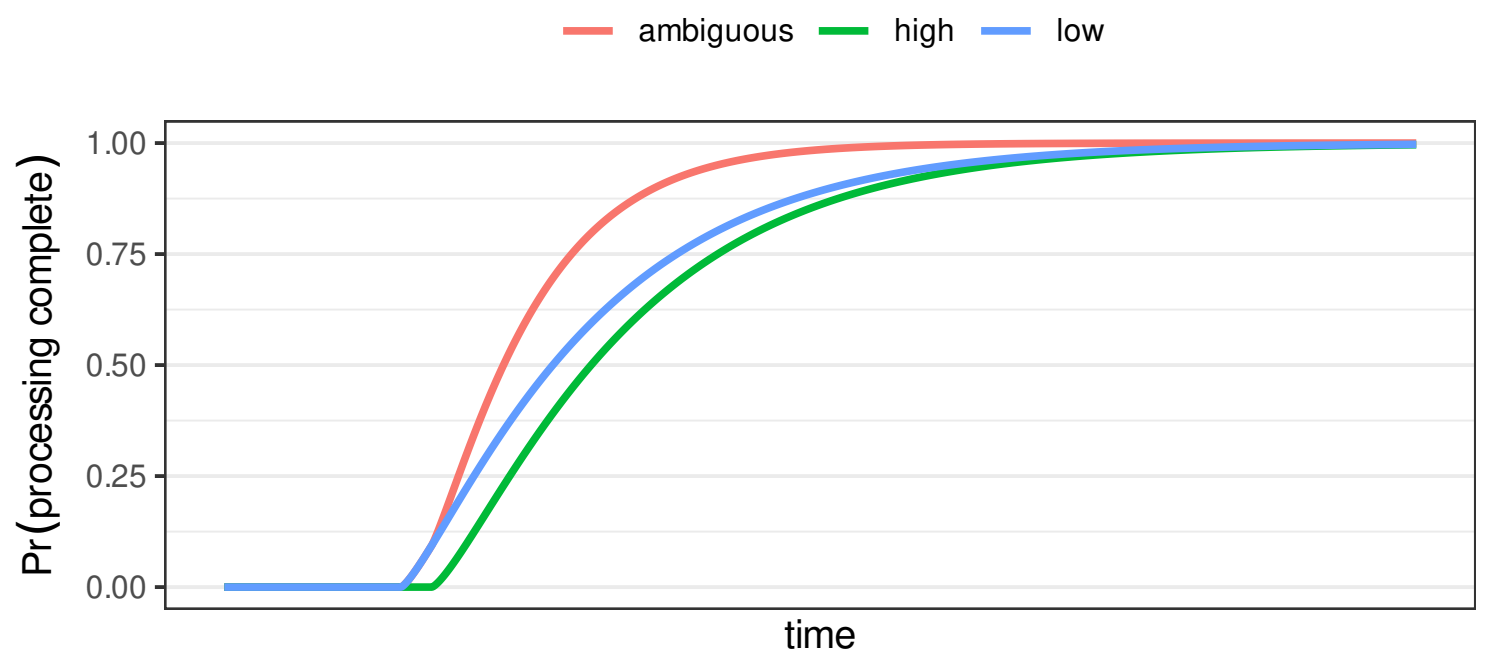

Figure 6

Probability of having successfully completed the $R C$ attachment at a particular time as predicted by unlimited-capacity race models. For expository reasons, the probability of successful completion of RC attachment is assumed to be 1 .

between ambiguous and unambiguous attachment conditions: As long as the probability of having completed either attachment is at zero, the probability of successfully completing the race is also at zero. $F_{A}$ departs from zero at the point in time when either $F_{L}$ or $F_{H}$ do. As both $F_{L}$ and $F_{H}$ increase, $F_{A}$ increases as well, albeit faster than either of the other completion time distributions, in accordance with equation 2.

$$
F_{A}(t)=F_{H}(t)+F_{L}(t)-F_{H}(t) \cdot F_{L}(t)
$$

For example, when the probability of a successful attachment being completed in unambiguous sentences is approximately 0.5 , it is 0.75 in the ambiguous condition. ${ }^{3}$ Because the fastest attachment times in the unambiguous conditions are also the fastest completion times in the ambiguous conditions, race models predict that the intercept of the SATF of the ambiguous condition should not differ from the intercept of the faster of the two intercepts of the unambiguous conditions. It is, however, predicted to be processed at a higher rate, which means that $\beta^{-1}$ (as illustrated in fig. 4) should be lower. It also follows from equation 2 that the asymptotic accuracy should be higher in the ambiguous condition than in its unambiguous counterparts unless both $\mathrm{RC}$ attachment processes are infallible and thus have a success probability of 1 .

\section{Limited-Capacity Race}

Although Lewis and Vasishth's (2005) cue-based parsing model is a type of race model (Nicenboim \& Vasishth, 2018), the probability of a successful retrieval does not follow

\footnotetext{
${ }^{3}$ This follows from equation 2.
} 
equation 2 because retrieval of both possible attachment sites is slowed due to cue-overload (e.g., Engelmann, Jäger, \& Vasishth, 2019) in the ambiguous condition. While in unambiguous conditions, a gender match between the relative pronoun and the $\mathrm{RC}$ attachment site uniquely identifies it, the retrieval cue gender matches two NPs in the the ambiguous condition. The result of this is the decreased uniqueness of the retrieval cue gender, which in turn slows the potential retrieval of either attachment site relative to its retrieval in the unambiguous conditions. The magnitude of the slowdown of the latent retrieval processes relative to their counterparts in unambiguous conditions depends on several ACT-R parameters, but its consequence is that the probability of having successfully retrieved any of the two potential attachment sites at any given point in time in the ambiguous condition is as in equation 3 , where $F_{H}^{\prime}$ and $F_{L}^{\prime}$ are the distribution functions of the slowed retrieval processes corresponding to high and low attachment. ${ }^{4}$ This relationship is illustrated in figure 7 , where $F_{H}^{\prime}$ and $F_{L}^{\prime}$ are represented by dotted lines.

$$
F_{A}(t)=F_{H}^{\prime}(t)+F_{L}^{\prime}(t)-F_{H}^{\prime}(t) \cdot F_{L}^{\prime}(t)
$$

Because ACT-R approximates the underlying retrieval time distributions $F_{L / H}$ and $F_{L / H}^{\prime}$ with unshifted log-normal distributions (Bothell, 2017; Nicenboim \& Vasishth, 2018) which have support on the entire positive part of the real line, the slowed retrieval will not affect the theoretical minimum retrieval times as they are always zero. However, if even the lowest percentiles of $F_{L / H}^{\prime}$ are substantially larger than the corresponding percentiles of $F_{L / H}$, approximating $F_{A}$ with equation (1) may result in larger intercept estimates in the ambiguous condition compared to the unambiguous conditions. Therefore, a limitedcapacity race such as the cue-based parsing account predicts either no difference in intercepts or a larger intercept in ambiguous conditions. Its predictions for rate and asymptote differences depend on the degree of slowdown due to cue-overload rendering the predictions for both highly dependent on a number of ACT-R parameters.

\section{Coactivation Models}

While Dillon et al. (2019) did not propose a specific process model of coactivation, a possible way to relate the idea of coactivation to timing is to conceptualize it as a sequential sampling model (e.g., Ratcliff et al., 2016) in which the sentence processor accumulates evidence for the existence of a valid parse for the current input. Figure 8 (lower panel) illustrates the increase in the amount of evidence for the existence of a valid parse as a function of time for all three attachment conditions (high, low and ambiguous) under the assumption that the amount of evidence $a_{A}$ in the ambiguous conditionat time $t$ is the sum of the evidence for the high attachment parse $a_{H}$ and the low attachment parse $a_{L}$, as in equation (4). Under the assumption that the speed of evidence accumulation varies from trial to trial following a normal distribution, the lines illustrate the average amount of activation at any given time, while the shaded regions show the trajectories within one standard deviation of the mean speed. Figure 8 (top panel) illustrates the corresponding distribution functions of completion times for all conditions under the assumption that a

\footnotetext{
${ }^{4}$ Due to the cue-overload related slowdown, $F_{H}$ and $F_{L}$ stochastically dominate $F_{H}^{\prime}$ and $F_{L}^{\prime}$ respectively, such that $F_{H}^{\prime}(t)<F_{H}(t)$ and $F_{L}^{\prime}(t)<F_{L}(t)$ for all $t$ such that $F_{L / H}(t)>0$ and $F_{L / H}^{\prime}(t)<1$.
} 


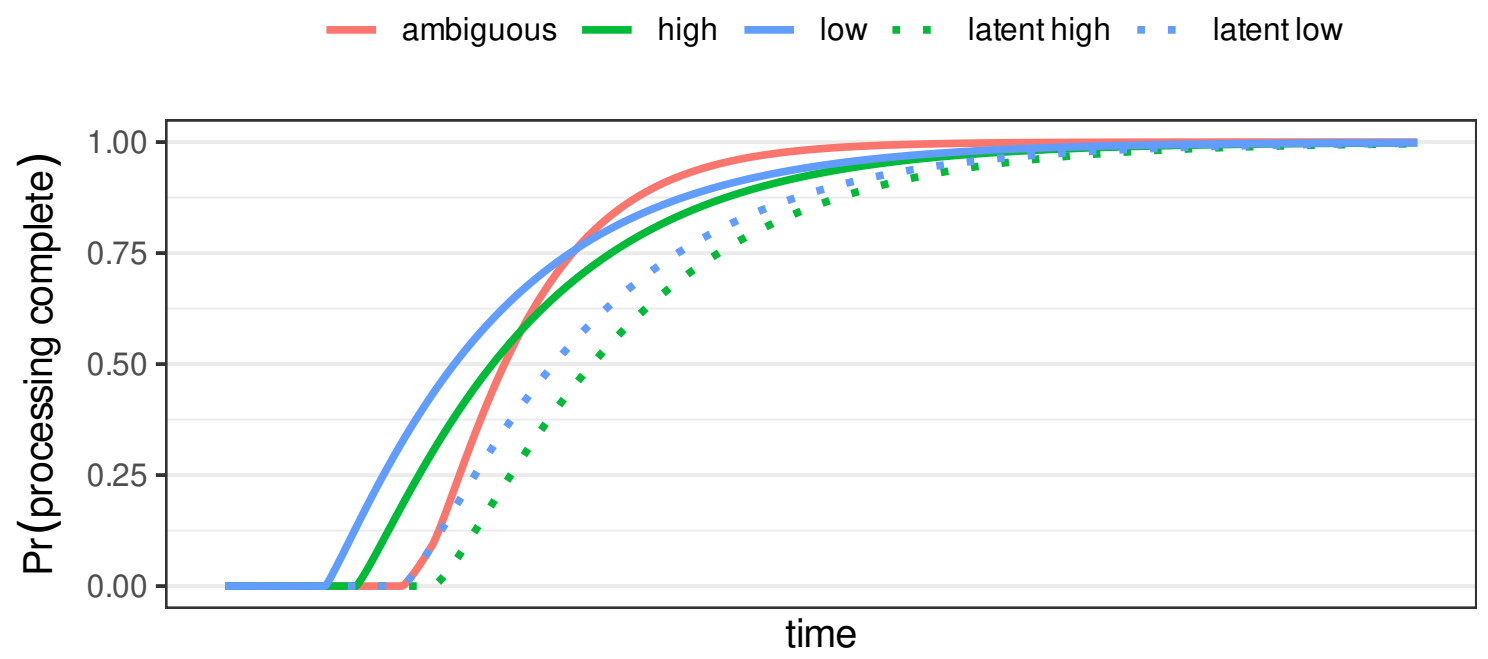

Figure 7

Probability of having successfully completed processing the $R C$ attachment at a particular time as predicted by a limited-capacity race model.

sufficient amount of evidence for the presence of a valid parse in condition $\mathrm{C}\left(a_{C}\right)$ has been obtained when $a_{C}$ exceeds a decision threshold as in equation (5).

As a result, the coactivation account predicts (i) shorter minimum processing times, (ii) a higher processing rate, and (iii) higher asymptotic accuracy in ambiguous sentences compared to their unambiguous counterparts.

$$
\begin{gathered}
a_{A}(t)=a_{H}(t)+a_{L}(t) \\
F_{C}(t)=\operatorname{Pr}\left(a_{C}(t)>\text { threshold }\right)
\end{gathered}
$$

\section{Summary}

Table 1 summarizes the predictions of the accounts discussed above. All accounts except limited capacity race models predict higher asymptotic accuracy $(\lambda)$ in the ambiguous condition than in the unambiguous conditions. ${ }^{5}$ Unlimited-capacity race models and coactivation models predict a lower $\beta^{-1}$ parameter (a higher rate) in the ambiguous condition, while the selective underspecification account predicts either a smaller $\beta^{-1}$ parameter parameter or a smaller intercept $(\delta)$ in the ambiguous condition. While unlimited-capacity race models predict equal intercepts in ambiguous and unambiguous conditions, limitedcapacity race models predict that intercepts in the ambiguous condition should be larger than or equal to the intercepts in unambiguous conditions. Meanwhile, coactivation models predict lower intercepts in ambiguous conditions.

\footnotetext{
${ }^{5}$ The cue-based parsing account is, in principle, even compatible with lower asymptotes in the ambiguous conditions under the assumption that the effect of cue-overload is implausibly large.
} 

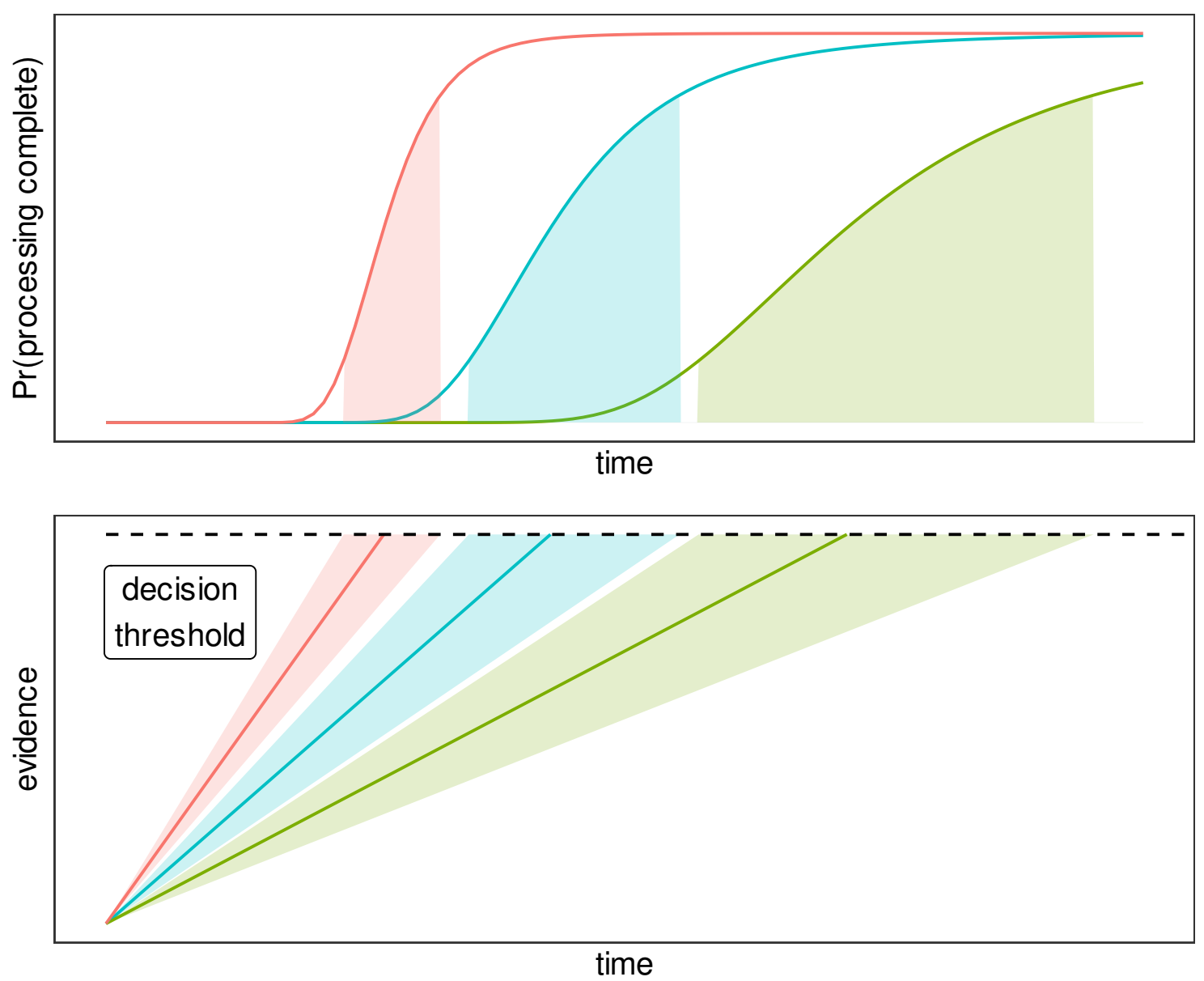

\section{Figure 8}

Probability of having successfully completed processing the $R C$ attachment at a particular time as predicted by a coactivation model (top panel) as a function of the total activation of the parses compatible with the current input string (bottom panel).

In sum, while there is a significant amount of overlap in the predictions, the intercept parameter is the most distinctive, because a lower intercept in the ambiguous condition is compatible only with the coactivation and the underspecification accounts, while a larger intercept is compatible only with limited-capacity race models. All models except for the coactivation account are compatible with the absence of a difference in intercepts, while unlimited-capacity race models specifically predict it. 


\begin{tabular}{rccc}
\hline & asymptote $(\lambda)$ & $1 /$ rate $\left(\beta^{-1}\right)$ & intercept $(\delta)$ \\
\hline Obligatory Underspecification & - & - & - \\
\hline Selective Underspecification & $\mathrm{A} \geq \mathrm{U}$ & $\mathrm{A}<\mathrm{U}$ & or $\quad \mathrm{A}<\mathrm{U}$ \\
\hline Coactivation models & $\mathrm{A}>\mathrm{U}$ & $\mathrm{A}<\mathrm{U}$ & $\mathrm{A}<\mathrm{U}$ \\
\hline Unlimited capacity race models & $\mathrm{A}>\mathrm{U}$ & $\mathrm{A}<\mathrm{U}$ & $\mathrm{A}=\mathrm{U}$ \\
\hline Limited capacity race models & & & $\mathrm{A} \geq \mathrm{U}$ \\
\hline
\end{tabular}

Table 1

Summary of the predictions the models make regarding the different SATF parameters. 'A' stands for ambiguous sentences. ' $U$ ' stands for unambiguous sentences. Dashes indicate compatibility with any result.

\section{Experiment 1}

\section{Method}

\section{Participants}

Twenty students from the University of Potsdam in the age range from 18 to 36 years participated in exchange for course credit. All were native speakers of German. All participants provided informed consent and the procedures in this study were compliant with the ethical principles outlined in the Helsinki Declaration on research involving human subjects.

\section{Procedure}

To measure participants' change in response accuracy over time in different attachment conditions, we used the multiple-response SAT procedure (MR-SAT; e.g., Dillon, 2011; Foraker, Cunnings, \& Martin, 2018; Foraker \& McElree, 2007; Franck \& Wagers, 2020; Kush, Johns, \& Van Dyke, 2019; Martin \& McElree, 2008, 2009, 2011; McElree, 1993; Van Dyke \& McElree, 2011; Wickelgren, Corbett, \& Dosher, 1980).

On each trial, a sentence was presented phrase by phrase in the center of the screen, as illustrated in figure 9. Each phrase was presented for $400 \mathrm{~ms}$, with an ISI of $50 \mathrm{~ms}$. A series of fourteen $500 \mathrm{~Hz}$ tones, with an SOA of $400 \mathrm{~ms}$ between tones started $600 \mathrm{~ms}$ before the onset of the last phase, comprising the entire relative clause. Each tone served as a cue for the participant to classify the sentence as acceptable or unacceptable by means of pressing a button on a game-pad. Participants were told that they did not need to (but were free to) respond to the first tone, as its main purpose was to serve as a preparatory cue. However, they were requested to respond to all tones following it. This is because early response cues in a sequence tend to be associated with an increased probability of missed responses and thus with less data for that time interval. To avoid substantial numbers of missed responses at short SOAs, our participants received two response cues before the onset of the RC (SOAs $-0.6 \mathrm{sec}$ and $-0.2 \mathrm{sec}$ ) in order to minimize the number of missing responses after the onset of the RC. Thus, the fist tone sounded $0.5 \mathrm{sec}$ before the presentation of the last phrase, while the last tone sounded at $4.6 \mathrm{sec}$ seconds thereafter.

One half of the participants was requested to press the right button with the index finger of their right hand to indicate the answer 'acceptable' while the other half were 


\section{Figure 9}

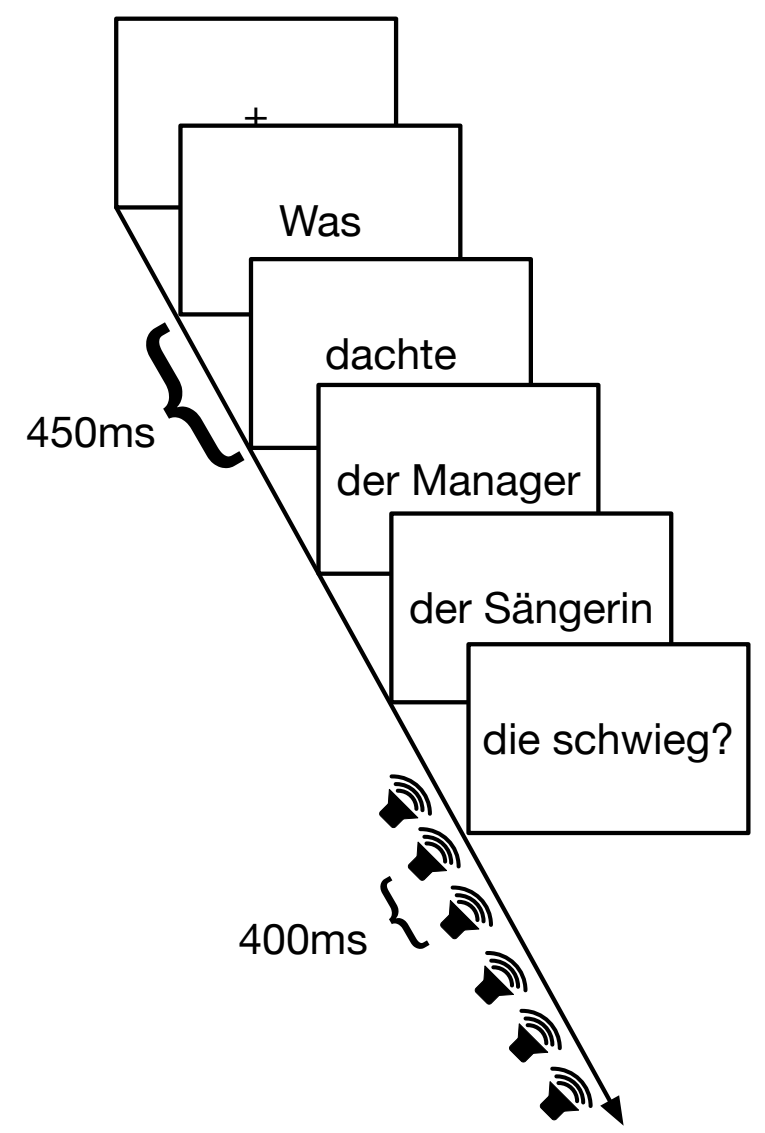

The structure of a SAT-trial in experiment 1. Sentences were presented phrase by phrase with an SOA of $450 \mathrm{~ms}$. Response signals began $600 \mathrm{~ms}$ before the onset of the last phrase (the relative clause).

requested to press this button to indicate the answer 'unacceptable.'

Before taking part in the experiment, participants practiced the task. First, they were trained to indicate the orientation of an arrowhead presented on the screen by means of pressing the left or the right button on a game-pad. This procedure served to familiarize participants with the pace at which they will have to press buttons during the experiment. Next, they were trained to modulate their responses based on changes in the arrowhead's direction during the trial. This training procedure consisted of 44 trials. Lastly, they practiced the actual experimental task on 67 unrelated sentences. Participants required approximately 30 minutes for the entire training session. The actual experiment took approximately 90 minutes excluding breaks and consisted of 16 blocks of 33 sentences each. Experimental sentences were presented along with 128 sentences from an unrelated experiment and 16 filler sentences which were always presented on the first trial of each of the 16 blocks. Participants were encouraged to take breaks between two blocks whenever they felt they needed one. 


\section{Stimuli}

We created 32 sets of sentences like (5). Each grammatical sentence from every set was presented to each participant once while each ungrammatical sentence was presented three times throughout the experiment in order to balance grammatical and ungrammatical sentences. We thus presented 192 grammatical experimental sentences (64 for each grammatical attachment condition) and 192 ungrammatical experimental items. The experimental sentences were intermixed with 144 additional sentences, of which one half was grammatical and the other half contained number agreement violations between subject and verb.

\section{a. HIGH, FEMININE RELATIVE PRONOUN}

Was dachte die Managerin des Sängers, die schwieg? What thought the manager.FEM of the singer.MASC, who.FEM was silent

b. HIGH, MASCULINE RELATIVE PRONOUN

Was dachte der Manager der Sängerin, der schwieg?

What thought the manager.MASC of the singer.FEM, who.MASC was silent

c. LOW, FEMININE RELATIVE PRONOUN

Was dachte der Manager der Sängerin, die schwieg?

What thought the manager.MASC of the singer.FEM, who.FEM was silent

d. LOW, MASCULINE RELATIVE PRONOUN

Was dachte die Managerin des Sängers, der schwieg?

What thought the manager.FEM of the singer.MASC, who.MASC was silent

e. AMBiguous, FEMININE RELATIVE PRONOUN

Was dachte die Managerin der Sängerin, die schwieg?

What thought the manager.FEM of the singer.FEM, who.FEM was silent

f. Ambiguous, MASCULINE RELATIVE PRONOUN

Was dachte der Manager des Sängers, der schwieg?

What thought the manager.MASC of the singer.MASC, who.MASC was silent

g. UNGRAMMATICAL, FEMININE RELATIVE PRONOUN

* Was dachte der Manager des Sängers, die schwieg?

What thought the manager.MASC of the singer.MASC, who.FEM was silent

'What did the manager of the singer who was silent think?'

h. UNGRAMMATICAL, MASCULINE RELATIVE PRONOUN

* Was dachte die Managerin der Sängerin, der schwieg?

What thought the manager.FEM of the singer.FEM, who.MASC was silent

'What did the manager of the singer who was silent think?'

In order to prevent participants from trying to detect and anticipate patterns in the presentation sequence and thus anticipating particular kinds of stimuli, an evolutionary algorithm (e.g., Eiben \& Smith, 2003) was used to create a pseudo-randomized stimulus list according to the following constraints: (1) We ensured that the grammaticality of a sentence could not be predicted from the grammaticality of the two preceding sentences. (2) The predictability of the experimental condition of the current sentence on the basis 
of the conditions of the two previous sentences was minimized. (3) The predictability of the experimental condition in which a particular item will occur next on the basis of the knowledge of its last two occurrences was kept as low as possible. (4) The probability that one item will regularly follow or precede another particular item was minimized as well. Given this set of constraints, we maximized the distance between repetitions of lexical material. We created one randomized list which half the participants saw in its regular order, while the other half saw it in the reverse order.

\section{Data Availability}

All data and code used in the data analysis is available at https://osf .io/z8tv9/.

\section{Data Preprocessing}

All data pre-processing and plotting was carried out in $R$ ( $\mathrm{R}$ Development Core Team, 2012) using the packages tidyverse and ggplot2 (Wickham et al., 2019). Two participants' data was excluded from further analysis, because they failed to consistently classify the low attachment condition as acceptable. Even at the latest lag, they judged low attachment sentences as acceptable on only $9 \%$ and $11 \%$ of the trials, respectively. All remaining participants judged all grammatical conditions as acceptable in at least $40 \%$ of the cases.

Exploratory data analysis showed substantial variation in the response times following the response signal, with systematic differences between participants and between lags. It also showed that some participants tended to respond multiple times per lag, suggesting that they may have attempted to repeatedly press the response button as quickly as possible rather than responding to the response signals. In order to ensure that each response signal corresponded to no more than one response, even if that response partially overlapped with the following response signal, we modelled the distribution of response times for each participants at each lag as a Gaussian with parameters to be estimated from the data using the Nelder and Mead (1965) numerical optimization algorithm. We used the resulting estimates to (i) re-bin the responses, and (ii) remove excess responses in each bin by keeping only those that were the closest to the average latency at that lag. Importantly, while only one response per bin was kept for the purposes of both plotting and statistical analysis, the association of responses with bins affected only the way in which $d^{\prime}$ was calculated for the purpose of plotting, and did not affect our statistical analysis, as it was conducted on individual responses and their respective response times.

\section{Statistical Analysis}

In order to correct for possible response bias towards 'acceptable' or 'unacceptable' responses, SAT data is typically analyzed through the lens of Signal Detection Theory (SDT) (e.g., Wickens, 2002). The key assumption of SDT when applied to acceptability judgements is that sentence acceptability is underlyingly continuous and that the acceptability of ungrammatical and grammatical sentences each follow a Gaussian distribution, with the distance between the means of the two distributions being equal to the sensitivity

$d^{\prime}$. When the perceived acceptability of a particular sentence falls below a response criterion $c$, the participant responds 'unacceptable'. When it is above $c$, they respond 'acceptable'. 
Because the sensitivity $d^{\prime}$ does not depend on the response criterion, it functions as a biasfree measure of response accuracy, and can be calculated from the percentages of responses corresponding to false alarms and hits following equation 6, where $z$ is the inverse Gaussian distribution function.

$$
d^{\prime}=z\left(p_{\text {hits }}\right)-z\left(p_{\text {false alarms }}\right)
$$

The analysis of data from SAT experiments typically involves model selection based on the goodness-of-fit of several models which are fit to each participant's data separately (e.g., Foraker et al., 2018; McElree \& Dosher, 1989). More specifically, $d^{\prime}$ is calculated for each participant in each experimental condition at each response lag, a range of models of varying complexity is fitted to each participant's data and the most parsimonious model for the entire dataset is selected on the basis of the results of by-participant fits. One problematic aspect of this method is that the uncertainty in the by-subject $d^{\prime}$ estimates at each lag is not taken into account. This is of particular importance because the sampling variability of $d^{\prime}$ generally increases with increasing accuracy, which means that data points closer to the asymptote tend to be associated with higher uncertainty than those in the earlier parts of the SAT function (e.g., Liu \& Smith, 2009). The problem is exacerbated by the fact that there are typically disproportionately many near-asymptotic data points, which are likely to be correlated in MR-SAT experiments. In consequence, the modelcomparison metric adjusted $R^{2}$ which is typically used in the analysis of SAT data places an undue emphasis on the part of the SATF with the highest sampling variability. The result is high variability of by-subject parameter estimates and thus relatively low statistical power even for modest effect sizes (Logačev \& Bozkurt, 2021; Pankratz, Yadav, Smith, \& Vasishth, 2021).

Hierarchical models (Gelman \& Hill, 2006) with the participants' responses as a dependent variable can be used to estimate the the sensitivity $d^{\prime}$ and the response criterion $c$ (e.g., DeCarlo, 1998; Wright \& London, 2009) while avoiding most of the above problems. This is because participant-level uncertainty is taken into account in the estimation of population-level effects. As a result of partial pooling, participant-level estimates associated with a high degree of uncertainty are shrunk towards the population mean. In consequence, population-level estimates with lower degrees of uncertainty exert the strongest influence on the population-level estimates.

In order to obtain more robust estimates of the parameters of interest, the experimental data was analyzed using a Bayesian hierarchical non-linear model with by-participant random effects implemented using the packages brms (Bürkner, 2017) and rstan (Stan Development Team, 2020). The model assumed that the probability of an 'acceptable' response at time $t$ followed equation 7 , where $d_{t}^{\prime}$ and $c_{t}$ are the sensitivity and response criterion at time $t$, and $\Psi$ is the Gaussian distribution function. The time of each response was determined relative to the presentation of the last phrase on the screen. Responses were not binned for the purposes of the statistical analysis.

$$
P\left(R_{t}=\text { 'acceptable' }\right)= \begin{cases}1-\Psi\left(c_{t}-d_{t}^{\prime}\right), & \text { in grammatical conditions } \\ 1-\Psi\left(c_{t}\right), & \text { in ungrammatical conditions }\end{cases}
$$




$\begin{array}{rccc}\text { parameter } & \text { transformation } & \text { intercept } & \text { slopes } \\ \lambda & \ln \lambda & N(0, .5) & N(0, .35) \\ \beta & \ln \beta^{-1} & N(0, .35) & N(0, .35) \\ \delta & \ln (\delta)-1 & N(0, .6) & N(0, .35) \\ \lambda_{C_{L}} & - & N(0,1.5) & - \\ \lambda_{C_{R}} & - & N(0,1.5) & N(0, .5) \\ \beta & \ln \beta_{C}^{-1} & N(0, .35) & N(0, .35) \\ \delta & \ln \left(\delta_{C}\right)-1 & N(0, .35) & N(0, .35)\end{array}$

Table 2

Prior distributions and transformation applied to each model parameter.

As is common in the SAT literature, the sensitivity $d^{\prime}$ at time $t$ was assumed to be a negatively accelerated shifted exponential function, as in equation 1 (repeated as 8). Here, $\lambda$ is the asymptotic sensitivity (in $d^{\prime}$ units), $\delta$ is the intercept of the SAT function in seconds (i.e., the time at which the sensitivity departs from zero), and $\beta$ is the rate of increase, which has a convenient interpretation as $\beta^{-1}$ (in seconds) as the amount of time the SAT function requires to reach approximately $63 \%$ of the asymptote.

$$
d_{t}^{\prime}=\lambda \cdot\left(1-e^{-\beta(t-\delta)}\right), \text { for } t \geq \delta ; 0 \text { otherwise }
$$

The response criterion was also assumed to be a function of time, following equation 9. Here, $\lambda_{c_{L}}$ is the initial response criterion, and $\lambda_{c_{R}}$ is the right asymptote of the response criterion function. $\beta_{c}$ is the rate of change and has the same interpretation as $\beta$ in equation 8. Finally, $\delta_{c}$ is the intercept of the response criterion function, and indicates the time at which the response criterion first departs from $\lambda_{c_{L}}$.

$$
c_{t}=\left\{\begin{array}{lr}
\lambda_{c_{L}}+\left(\lambda_{c_{R}}-\lambda_{c_{L}}\right) \cdot\left(1-e^{-\beta_{c}\left(t-\delta_{c}\right)}\right), & \text { for } t \geq \delta_{c} \\
\lambda_{c_{L}}, & \text { otherwise }
\end{array}\right.
$$

The model was fitted with the following orthogonal contrasts for all three parameters of the $d^{\prime}$ function: (i) relative pronoun gender (masculine-feminine), (ii) RC attachment (high attachment - average and low attachment - average), and (iii) the interactions between the previous two. ${ }^{6}$ The only contrast specified for the response criterion was relative pronoun gender. We used the maximal by-subject random effect structure for all model parameters $\left(\lambda, \beta^{-1}, \delta, \lambda_{C_{L}}, \lambda_{C_{R}}, \beta_{C}^{-1}, \delta_{C}\right)$, including correlations between all coefficients within each SATF parameter. The priors and parameter transformations that were used in the model are specified in table 2 .

Four sampling chains were run, with 4000 iterations each, after a burn-in of 1000 iterations. While all coefficients were estimated on log-scale, the model's posterior MCMC (Markov-Chain Monte Carlo) samples were used to compute credible intervals and posterior probabilities on the original scale (seconds and $d^{\prime}$ units). Coefficient estimates will be

\footnotetext{
${ }^{6}$ In the model, the effect of RC attachment was represented using sum contrasts (high attachment - average and low attachment - average), and was later re-coded to the equivalent of sliding differences contrasts (high attachment - ambiguous and ambiguous - low attachment) with the latter contrast inverted to low attachment - ambiguous for better interpretability of the coefficient estimates.
} 
presented along with $90 \%$ and $80 \%$ credible intervals. The posterior probability that a parameter is smaller than (or larger than) zero is above $90 \%$ if the $80 \%$ credible interval excludes zero, and larger than $95 \%$ if the $90 \%$ credible interval excludes zero. In addition to the credible intervals, the posterior probability of a particular parameter $\theta$ being smaller or larger than zero was computed where required.

A potential problem for this analysis method is the fact that successive responses on one trial are likely to be correlated in MR-SAT. This serial correlation is not accounted for in the method used here, because doing so would require making use of an approximation to the bivariate normal cumulative distribution function (e.g., Albers \& Kallenberg, 1994; Pan, 2017; Tsay \& Ke, 2021), which tend to be computationally intensive and numerically unstable. As a result, responses are modelled as independent, although it is currently not clear how or whether this may affect the results. Importantly, this simplification does not constitute a drawback of the present analysis method, since more commonly used methods for the analysis of MR-SAT (e.g., Foraker et al., 2018) data do not account for the correlation of successive responses.

\section{Results}

Figure 10 shows the average $d^{\prime}$ by attachment condition at different lags along with the model predictions. At later lags, ambiguous conditions are responded to more accurately than both unambiguous conditions, with no appreciable differences between low and high attachment conditions.

A non-linear hierarchical Bayesian model of the speed-accuracy tradeoff function in equation 8 was fitted to the participants' responses. All $\hat{R}$ estimates were 1.00 , indicating model convergence. The estimated effective sample size $\left(E S S_{\text {bulk }}\right.$ and $\left.E S S_{\text {tail }}\right)$ was larger than 4,000 for all $\delta$ and $\beta^{-1}$ coefficients, larger than 2,500 for all $\lambda$ coefficients except for the average $\lambda$, for which $E S S_{\text {bulk }}=967$ and $E S S_{\text {tail }}=2,096$. Figure 11 shows the $90 \%$ and $80 \%$ credible intervals of the non-linear hierarchical Bayesian model of the speedaccuracy tradeoff functions according to equation 8 . Its upper panels show that the average intercept $(\delta)$ was in the range $[0.35 ; 0.46] \mathrm{sec}$, the average reciprocal rate $\left(\beta^{-1}\right)$ was in the range $[0.53 ; 0.72] \mathrm{sec}$, while the average asymptote $(\lambda)$ was in the range $[2.04 ; 2.85]$.

The lower panels of figure 11 show the estimates of the effects of the experimental manipulation on the parameters of the speed-accuracy tradeoff function. ${ }^{7}$ a smaller $S A T F$ intercept in the ambiguous attachment condition than in the low attachment condition $(\mathrm{CrI}=[-0.14 ; 0.00], P(\theta<0)=0.947)$ or the high attachment condition $(C r I=[-0.12 ; 0.00], P(\theta<0)=0.944)$. In addition to a lower SATF intercept, ambiguous RC attachment was associated with a higher asymptotic accuracy than either low attachment $(C r I=[0.52 ; 1.32], P(\theta<0)<0.001)$ or high attachment $(C r I=[0.69 ; 1.52], P(\theta<0)<0.001)$. Attachment did not appear to have an unambiguous effect on the $\beta^{-1}$ parameter (1/rate), although processing appears to have been somewhat slower in the ambiguous conditions relative to their unambiguous counterparts (low attachment: $C r I=[-0.06 ; 0.20], P(\theta<0)=0.182$; high attachment: $C r I=[-0.01 ; 0.24]$, $P(\theta<0)=0.078)$. Moreover, the analysis showed a main effect of relative pronoun

\footnotetext{
${ }^{7}$ Figure A2 in the appendix shows the corresponding by-participant estimates of the same parameters based on the hierarchical model. The coefficient estimates in it indicate
} 


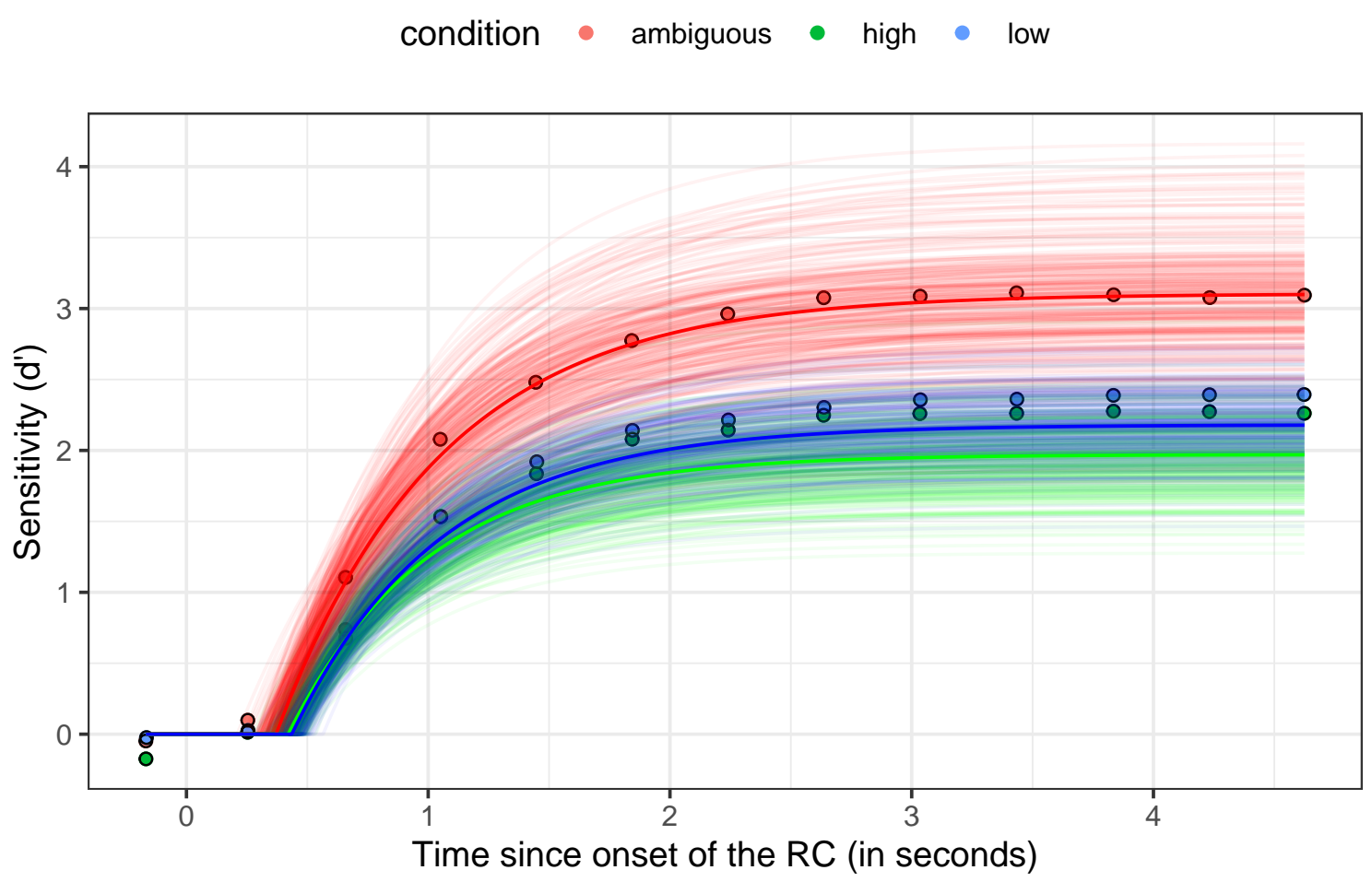

Figure 10

Average d' at different lags after the presentation of the two-word relative clause (circles). Model predictions based on fixed effects maximum a-posteriori (MAP) estimates (solid lines), along with a spaghetti plot illustrating the uncertainty in the model predictions based on 250 random samples from the model (semi-transparent lines).

gender, indicating that masculine relative pronoun conditions were associated with higher intercepts $(\mathrm{CrI}=[0.01 ; 0.14], P(\theta<0)=0.028)$, as well as somewhat lower asymptotic accuracy $(C r I=[-0.61 ;-0.15], P(\theta<0)=0.996)$.

The only noteworthy interaction between gender and attachment affected asymptotic accuracy, indicating larger asymptotic accuracy differences between ambiguous and unambiguous sentences in masculine relative pronoun conditions (high attachment: $\mathrm{CrI}=$ [0.51; 1.31], $P(\theta<0)<0.001$ ), (low attachment: $C r I=[0.02 ; 0.82], P(\theta<0)=0.040$ ). There was weak evidence for an interaction in $S A T F$ intercepts suggesting a larger (negative) intercept difference between ambiguous and high attachment sentences in masculine relative pronoun conditions (high attachment: $C r I=[-0.16 ; 0.26], P(\theta<0)=0.337$ ), as well as an attenuation of the intercept difference between ambiguous and low attachment conditions $\operatorname{Cr} I=[-0.20 ; 0.23], P(\theta<0)=0.455)$,

\section{Discussion}

The main finding in this experiment was that the SATF of sentences with ambiguous relative clause attachment is characterized by higher asymptotes and lower intercepts than 

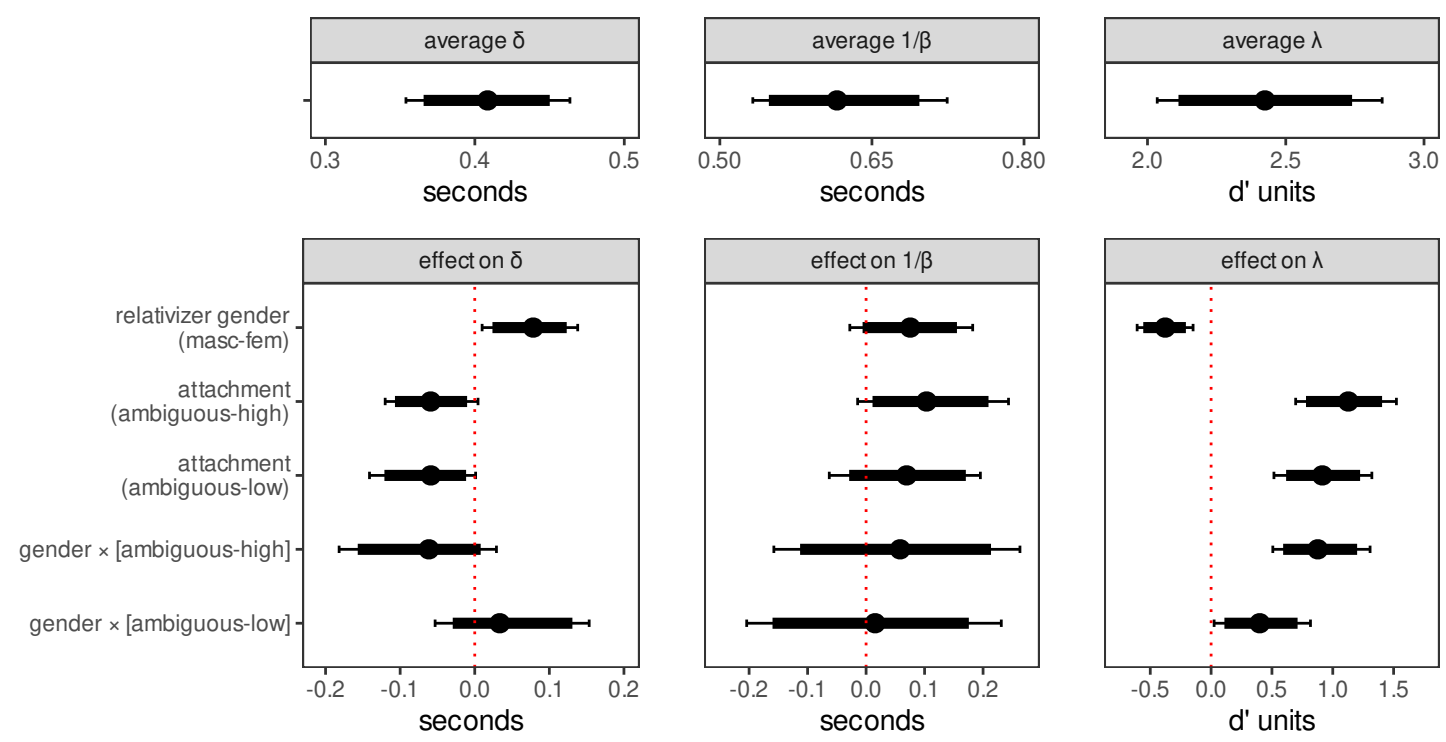

Figure 11

Credible intervals for the posterior distributions of the coefficients of a non-linear hierarchical Bayesian model, by speed-accuracy trade-off function parameter (intercept, 1/rate, asymptote). Thin lines represent $90 \%$ CrIs and thick lines represent $80 \%$ CrIs.

the SATFs of either of the unambiguous conditions. This means that ambiguous sentences have (i) a higher probability of successful processing and (ii) shorter minimal processing times compared to their unambiguous counterparts.

The latter finding is incompatible with either class of race models discussed here because both unlimited-capacity models (SMCM and URM) predict no differences in intercept, while the limited-capacity cue-based retrieval account predicts either no difference in intercept, or larger minimum processing times in ambiguous conditions. Moreover, unlimited-capacity race models predict a higher processing rate in ambiguous conditions, compared to their unambiguous counterparts, and while our findings regarding rates are not unambiguous in that they appear to be compatible with lower rates in ambiguous conditions or no differences in rates, they do not suggest a higher rate of processing in the ambiguous condition than in either unambiguous condition. Although the proposed mechanism for a coactivation model is compatible with both lower intercepts, and higher asymptotes in ambiguous conditions, and while the present findings are compatible with a small increase in rates, they don't provide any evidence for one. Both underspecification accounts are compatible with the findings because selective underspecification predicts shorter intercepts in the ambiguous condition compared to the unambiguous condition while the obligatory underspecification account is compatible with any outcome. The higher asymptotic accuracy in the ambiguous condition is compatible with all current accounts of the ambiguity advantage.

In addition to the main effects of attachment, we observed an interaction between attachment and relative pronoun gender, which indicated larger asymptotic accuracy differences between ambiguous and unambiguous sentences in masculine relative pronoun con- 
ditions than in the feminine relative pronoun conditions. This interaction is likely due to a local ambiguity in the masculine relative pronoun conditions: The German singular relative pronoun 'der', which was used to disambiguate attachment towards a masculine noun phrase is ambiguous between nominative masculine and dative feminine. While it is clearly disambiguated towards the masculine nominative by the fact that it is the only argument of a verb in the active voice, this disambiguation occurs at the second word of the relative clause. Although in German, nominative relative pronouns are significantly more frequent than dative relative pronouns (e.g., Logačev \& Vasishth, 2012), it is possible that the parser sometimes entertains a feminine-dative interpretation of the relative pronoun. In consequence, it may attempt to form a structure involving the (incorrect) attachment of the $\mathrm{RC}$ to a feminine noun phrase when one is present and a failure to integrate a verb in active voice with no further arguments into a structure with a dative relativizer. As a result, processing may occasionally be impaired in the unambiguous conditions with a masculine relative pronoun resulting in lower asymptotes in those conditions, and thus in larger asymptote differences between ambiguous and unambiguous conditions with masculine relative pronouns. It is moreover possible that the effect is more pronounced in high attachment conditions than in low attachment conditions because the locally ambiguous relative pronoun is adjacent to the feminine noun phrase in the high attachment condition with a masculine relative pronoun $(5 \mathrm{~b})$, but not in $(5 \mathrm{~d})$, its low attachment counterpart. The effect of reanalysis due to local ambiguity may also explain the weak interaction between gender and attachment (ambiguous-high) in intercept $\delta$, as effects of reanalysis on dynamics parameters have previously been attested (Bornkessel, McElree, Schlesewsky, \& Friederici, 2004; Martin \& McElree, 2018).

A common concern in the analysis of SAT data is that the estimates of the dynamics parameters $\beta^{-1}$ and $\delta$ in equation 8 tend to be correlated in that a decrease in the function's goodness of fit due to a lower rate $\delta$ can (partially) be compensated for by an increase in $\beta^{-1}$, and vice versa (e.g., Foraker et al., 2018). This is largely due to the fact that both parameters exert their greatest influence by jointly accounting for a relatively small number of response lags around the time when the SATF shows the largest amount of change (between $0 \mathrm{sec}$ and $2 \mathrm{sec}$ in the present experiment). This is in contrast to the asymptote $\lambda$ which, with a relatively small influence from both dynamics parameters, accounts for most of the variance in the responses from $2 \mathrm{sec}$ to $4.5 \mathrm{sec}$. Importantly, the correlation between $\beta^{-1}$ and $\delta$ may have two effects: Firstly, the shape of their joint likelihood function may complicate sampling or estimation as a result of the geometry of their joint likelihood function (e.g., Nicenboim, Schad, \& Vasishth, 2021). Secondly, a strong correlation between two parameters in the posterior may indicate parameter unidentifiability or weak identifiability, and thus a model overparameterization (e.g., Gimenez, Morgan, \& Brooks, 2009). These issues will be addressed in turn.

While the correlation between the average intercept and the average rate parameters was relatively weak $(r=-0.2)$, all other coefficients exhibited a moderate amount of correlation $(r \approx-0.5)$. Figure A3 in the appendix shows the correlation between posterior samples of the rate and intercept coefficients. Importantly, the estimated effective sample size $\left(E S S_{\text {bulk }}\right.$ and $\left.E S S_{\text {tail }}\right)$ indicated a sufficient number of posterior samples required for inference, which along with an $\hat{R}$ of 1.00 indicated no problems during the sampling process.

However, the main parameter of interest (the effect of attachment on the intercept) 
may still be weakly unidentifiable; Gimenez et al. (2009) define a parameter as unidentifiable if more than one parameter value corresponds to the model's maximum likelihood for the data. This can happen in overparameterized models, when a decrease in a model's goodness of fit due to the change in one parameter can be compensated for by a corresponding change in another parameter. As a result, the parameter's posterior distribution is equal to its prior distribution. A related notion is weak unidentifiability, which arises when the data supply little information about one or multiple parameters. As a result, the posterior distribution of a weakly identifiable parameter is approximately equal to its prior distribution (Garrett \& Zeger, 2000). An informal comparison between the prior distributions of the coefficients in Figure A1 in the appendix, and their posterior distributions in Figure 11 shows that posterior and prior distributions are far from being approximately equal. Garrett and Zeger (2000) propose the metric $\tau$ in equation 10 as a formal measure of overlap between posterior and prior. $\tau$ is in the range from 0 to 1 and can be interpreted as the amount of overlap between the prior and the posterior distribution. They suggest to interpret a $\tau$ of 0.35 or more as an indicator of weak identifiability (cf. Garrett \& Zeger, 2000; Gimenez et al., 2009, for a discussion). We calculated $\tau$ for all fixed effects in our model and found that $\tau<0.35$ for all $d^{\prime}$-related parameters with the exception of all effects involving gender for the intercept $\delta(\tau \approx 0.4)$, and the reciprocal rate $\beta^{-1}(0.44 \leq \tau \leq 0.5)$. In sum, it appears that all coefficients for the main effect of attachment were identifiable, indicating that the relatively low correlations between rate and intercept did not affect statistical inference.

$$
\tau=\int \min (p(\theta), \pi(\theta \mid Y)) \cdot d \theta
$$

\section{General Discussion}

The aim of the present study was to test the predictions of several competing accounts of the ambiguity advantage. While all accounts predict faster processing in ambiguous sentences compared to their unambiguous counterparts, they differ in their assumptions about the mechanisms that effect that speedup. As a result, they differ in their predictions regarding the completion time distributions of the attachment process in different attachment conditions, and thus in their predictions about differences in the speed-accuracy tradeoff functions (SATFs) in ambiguous and unambiguous conditions. Limited-capacity race models such as the URM and SMCM (Logačev \& Vasishth, 2016; van Gompel et al., 2001) predict no differences in minimum completion times between ambiguous and unambiguous conditions, and thus between their SATF intercepts. Meanwhile, the underspecification account (Swets et al., 2008) and the class of limited-capacity race models are less definitive in their predictions. While the underspecification account is compatible with lower minimum processing times in ambiguous sentences, the unlimited-capacity race account predicts higher or equal minimum processing times. As a result, the underspecification account predicts either no difference in intercepts, or a smaller intercept in the ambiguous conditions, while limited-capacity race models predict larger or equal intercepts in the ambiguous condition. While the class of possible coactivation accounts in general may differ in their specifics, the particular implementation of the coactivation account proposed here predicts lower intercepts and higher rates and asymptotes in ambiguous conditions.

An MR-SAT experiment in German provided evidence for (i) higher asymptotic 
accuracy and (ii) lower SATF intercepts in ambiguous sentences compared to their unambiguous counterparts. While the former finding is expected under all models considered here, the latter finding is not compatible with limited-capacity or unlimited-capacity race models because they predict either no difference in minimum processing times or larger minimum processing times in ambiguous structures. The only accounts compatible with the observed difference in intercepts are underspecification and the coactivation account.

Importantly, there are two possible instantiations of the underspecification account: The obligatory underspecification account, assumes that underspecification is always carried out at the relative pronoun if it does not disambiguate $\mathrm{RC}$ attachment, and therefore always precedes full RC attachment, as in examples (1), (2), and (3). In consequence, attachment is only carried out upon disambiguation of the sentence. In contrast to selective underspecification, the obligatory underspecification account makes no clear predictions for sentences like (4) which were used in the experiment because its predictions depend on assumptions about the relative durations of underspecification of $\mathrm{RC}$ attachment on the one hand and full RC attachment on the other. Thus, while it is compatible with the findings presented here under the right assumptions about process durations, it fails to account for the Dillon et al. (2019) data, because unlike the stimuli in the present experiment, the sentences in (3) were disambiguated at the second word, which means that according to obligatory underspecification, all conditions should have been underspecified initially. Since the task demands in Dillon et al.'s experiment never required disambiguation, the obligatory underspecification account predicts that the underspecified representation could have been used to correctly judge acceptable sentences as acceptable in all attachment conditions with no between-condition differences in timing or accuracy. Thus, Dillon et al.'s findings provide evidence against the obligatory underspecification account.

The selective underspecification account, on the other hand, assumes that RC attachment is postponed until the point in the sentence where the parser can plausibly use all available information to resolve a structural ambiguity, which is when the parser decides whether to underspecify or to attach the RC. Under this account, a difference in minimum processing times between ambiguous and unambiguous conditions can be explained under the assumption that underspecification is not only faster on average than full RC attachment, but also has shorter minimum completion times.

While the underspecification account may appear to match the SAT findings more closely than the coactivation in that it is compatible with the absence of a difference in processing rates, the absence of evidence for such a difference does not necessarily constitute evidence against it. This is because firstly, our findings are compatible with a higher rate (smaller $\beta^{-1}$ ) in the ambiguous condition, although this effect is likely to be relatively small. Secondly, the magnitude of the predicted difference in processing rates is difficult to quantify without a computational implementation of the coactivation model.

In consequence, both the selective underspecification and the coactivation model can account for a range of ambiguity-advantage related findings, including the presence of an ambiguity advantage in natural reading (Traxler et al., 1998; Van Gompel et al., 2005; van Gompel et al., 2001) and speeded-acceptability judgements (Dillon et al., 2019), as well as in SATF intercepts in the present study. It remains to be determined to what extent the coactivation model can account for the potential task-dependence of the underspecification effect argued for by Swets et al. (2008). 
Importantly, both accounts currently require ad hoc assumptions in order to account for those findings. Specifically, the coactivation account needs to assume a small difference in rates between ambiguous and unambiguous sentences in order to account for the findings in the present experiment. The underspecification account, on the other hand, needs to stipulate that the underspecification of RC attachment is not only faster than full $\mathrm{RC}$ attachment on average, but also that its minimum duration is shorter. Moreover, it needs to assume that underspecification of RC attachment has a higher probability of being executed successfully. A further non-trivial assumption of the underspecification account is that unambiguous sentences cannot be underspecified. While that is unsurprising on the surface, as an underspecified structure cannot constitute a verdical representation of an unambiguous sentence, such a representation is arguably 'good enough' for a range of tasks (e.g., Ferreira, Bailey, \& Ferraro, 2002) which begs the question of why a 'good enough' parser is unable to make use of this operation on unambiguous sentences. At present, these assumptions do not appear to be independently motivated. However, they may ultimately follow from independent principles. For instance, it is possible that underspecified syntactic representations are not semantically interpreted, in which case both timing assumptions of the selective underspecification account follow from the presence of an additional process in the unambiguous conditions, while the lower asymptotic accuracy in the unambiguous conditions may follow from the fact that semantically interpreted unambiguous sentences can be rejected for extra-syntactic reasons, while the uninterpreted ambiguous sentences cannot. Further theoretical work is needed to determine determined how exactly underspecified or coactive structures are represented and to what extent the notions of underspecification or coactivation is compatible with models such as surprisal (Hale, 2001; Levy, 2008) or the self-organized sentence account (e.g., Cho, Goldrick, \& Smolensky, 2020; Smith, 2018; Tabor \& Hutchins, 2004).

\section{References}

Albers, W., \& Kallenberg, W. C. (1994). A simple approximation to the bivariate normal distribution with large correlation coefficient. Journal of multivariate analysis, 49(1), $87-96$.

Anderson, J. R., Bothell, D., Byrne, M. D., Douglass, S., Lebiere, C., \& Qin, Y. (2004). An integrated theory of the mind. Psychological review, 111(4), 1036.

Anderson, J. R., \& Reder, L. M. (1999). The fan effect: New results and new theories. Journal of Experimental Psychology: General, 128(2), 186.

Bader, M., \& Häussler, J. (2010). Toward a model of grammaticality judgments. Journal of Linguistics, 46(2), 273-330. doi: 10.1017/S0022226709990260

Bornkessel, I., McElree, B., Schlesewsky, M., \& Friederici, A. D. (2004). Multi-dimensional contributions to garden path strength: Dissociating phrase structure from case marking. Journal of Memory and Language, 51(4), 495-522.

Bothell, D. (2017). Act-r 7 reference manual. Available at act-r psy cmu edu/wordpress/wpcontent/themes/ACT-R/actr7/reference-manual pdf, Accessed February.

Bürkner, P.-C. (2017). brms: An r package for bayesian multilevel models using stan. Journal of Statistical Software, 1(80), 1-28. doi: 10.18637/jss.v080.i01 
Cho, P. W., Goldrick, M., \& Smolensky, P. (2020). Parallel parsing in a gradient symbolic computation parser.

Christianson, K., Hollingworth, A., Halliwell, J. F., \& Ferreira, F. (2001). Thematic Roles Assigned along the Garden Path Linger. Cognitive Psychology, 42(4), 368-407. doi: 10.1006/cogp.2001.0752

DeCarlo, L. T. (1998). Signal detection theory and generalized linear models. Psychological methods, 3(2), 186.

Dillon, B. (2011). Structured access in sentence comprehension (Unpublished doctoral dissertation). University of Maryland, College Park.

Dillon, B., Andrews, C., Rotello, C. M., \& Wagers, M. (2019). A new argument for coactive parses during language comprehension. Journal of Experimental Psychology: Learning, Memory, and Cognition, 45(7), 1271.

Dillon, B., \& Wagers, M. (2019). Approaching gradience in acceptability with the tools of signal detection theory.

Dosher, B. (1976). The Retrieval of Sentences From Memory: A Speed-Accuracy Study. Cognitive Psychology, 310, 291-310.

Eiben, A. E., \& Smith, J. E. (2003). Introduction to evolutionary computing (Vol. 53). Springer.

Engelmann, F., Jäger, L. A., \& Vasishth, S. (2019). The effect of prominence and cue association on retrieval processes: A computational account. Cognitive Science, 43(12), e12800.

Ferreira, F. (2003). The misinterpretation of noncanonical sentences. Cognitive Psychology, $47(2), 164-203$.

Ferreira, F., Bailey, K. G., \& Ferraro, V. (2002). Good-enough representations in language comprehension. Current directions in psychological science, 11(1), 11-15.

Foraker, S., Cunnings, I., \& Martin, A. E. (2018). Speed-accuracy tradeoff modeling and its interface with experimental syntax.

Foraker, S., \& McElree, B. (2007). The role of prominence in pronoun resolution: Active versus passive representations. Journal of Memory and Language, 56(3), 357-383. doi: $10.1016 / j . j m l .2006 .07 .004$

Franck, J., \& Wagers, M. (2020). Hierarchical structure and memory mechanisms in agreement attraction. PLOS ONE, 15(5), e0232163. doi: 10.1371/journal.pone.0232163

Frazier, L., \& Rayner, K. (1982). Making and correcting errors during sentence comprehension: Eye movements in the analysis of structurally ambiguous sentences. Cognitive Psychology, 14 (2), 178-210. doi: 10.1016/0010-0285(82)90008-1

Garrett, E. S., \& Zeger, S. L. (2000). Latent class model diagnosis. Biometrics, 56(4), $1055-1067$.

Gelman, A., \& Hill, J. (2006). Data analysis using regression and multilevel/hierarchical models. Cambridge university press.

Gimenez, O., Morgan, B. J., \& Brooks, S. P. (2009). Weak identifiability in models for markrecapture-recovery data. In Modeling demographic processes in marked populations (pp. 1055-1067). Springer.

Green, M. J., \& Mitchell, D. C. (2006). Absence of real evidence against competition during syntactic ambiguity resolution. Journal of Memory and Language, 55(1), 1-17.

Hale, J. (2001). A probabilistic earley parser as a psycholinguistic model. In Proceed- 
ings of naacl 2001 (pp. 1-8). Morristown, NJ, USA: Association for Computational Linguistics.

Kush, D., Johns, C. L., \& Van Dyke, J. A. (2019). Prominence-sensitive pronoun resolution: New evidence from the speed-accuracy tradeoff procedure. Journal of Experimental Psychology: Learning, Memory, and Cognition, 45(7), 1234-1251. doi: 10.1037/ $\mathrm{xlm} 0000646$

Levy, R. (2008). Expectation-based syntactic comprehension. Cognition, 106(3), 11261177. doi: 10.1016/j.cognition.2007.05.006

Lewis, R. L. (2000). Falsifying Serial and Parallel Parsing Models: Empirical Conundrums and An Overlooked Paradigm. Journal of Psycholinguistic Research, 29(2), 8.

Lewis, R. L., \& Vasishth, S. (2005). An Activation-Based Model of Sentence Processing as Skilled Memory Retrieval. Cognitive Science, 29(3), 375-419. doi: $10.1207 / \mathrm{s} 15516709 \operatorname{cog} 0000 \_25$

Liu, C. C., \& Smith, P. L. (2009). Comparing time-accuracy curves: Beyond goodnessof-fit measures. Psychonomic Bulletin \& Review, 16(1), 190-203. doi: 10.3758/ PBR.16.1.190

Logačev, P., \& Bozkurt, M. I. (2021). Statistical power in response signal paradigm experiments. In Proceedings of the 43rd annual conference of thecognitive science society (pp. 39-47).

Logačev, P., \& Vasishth, S. (2012). Case matching and conflicting bindings interference. In Case, word order and prominence (pp. 187-216). Springer.

Logačev, P., \& Vasishth, S. (2016). A multiple-channel model of task-dependent ambiguity resolution in sentence comprehension. Cognitive Science, 40(2), 266-298.

Martin, A. E., \& McElree, B. (2008). A content-addressable pointer mechanism underlies comprehension of verb-phrase ellipsis. Journal of Memory and Language, 58(3), 879906.

Martin, A. E., \& McElree, B. (2009). Memory operations that support language comprehension: Evidence from verb-phrase ellipsis. Journal of Experimental Psychology: Learning, Memory, and Cognition, 35(5), 1231-1239. doi: 10.1037/a0016271

Martin, A. E., \& McElree, B. (2011). Direct-access retrieval during sentence comprehension: Evidence from Sluicing. Journal of Memory and Language, 64(4), 327-343. doi: 10.1016/j.jml.2010.12.006

Martin, A. E., \& McElree, B. (2018). Retrieval cues and syntactic ambiguity resolution: speed-accuracy tradeoff evidence. Language, cognition and neuroscience, 33(6), 769783.

McElree, B. (1993). The Locus of Lexical Preference Effects in Sentence Comprehension: A Time-Course Analysis. Journal of Memory and Language, 32(4), 536-571. doi: 10.1006/jmla.1993.1028

McElree, B., \& Dosher, B. A. (1989). Serial position and set size in short-term memory: the time course of recognition. Journal of Experimental Psychology: General, 118(4), 346.

McRae, K., Spivey-Knowlton, M. J., \& Tanenhaus, M. K. (1998). Modeling the Influence of Thematic Fit (and Other Constraints) in On-line Sentence Comprehension. Journal of Memory and Language, 38(3), 283-312. doi: 10.1006/jmla.1997.2543

Miller, J. (1982). Divided attention: Evidence for coactivation with redundant signals. 
Cognitive psychology, 14(2), 247-279.

Nelder, J. A., \& Mead, R. (1965). A simplex method for function minimization. The computer journal, 7(4), 308-313.

Nicenboim, B., Schad, D. J., \& Vasishth, S. (2021). An introduction to bayesian data analysis for cognitive science. Retrieved from https://vasishth.github.io/bayescogsci/ book/

Nicenboim, B., \& Vasishth, S. (2018). Models of retrieval in sentence comprehension: A computational evaluation using bayesian hierarchical modeling. Journal of Memory and Language, 99, 1-34.

Pachella, R. (1974). The Interpretation of Reaction Time in Information Processing Research. In B. H. Kantowitz (Ed.), Human information processing: Tutorials in performance and cognition. Hillsdale, N.J.: Erlbaum Associates.

Pan, K. (2017). An analytical expression for bivariate normal distribution. Available at SSRN 2924071.

Pankratz, E., Yadav, H., Smith, G., \& Vasishth, S. (2021). Statistical properties of the speed-accuracy trade-off (sat) paradigm in sentence processing. In Proceedings of the annual meeting of the cognitive science society (Vol. 43).

Pinheiro, J. C., \& Bates, D. M. (2000). Mixed-effects models in s and s-plus. Statistics and Computing.

R Development Core Team. (2012). R: A Language and Environment for Statistical Computing. Vienna, Austria: R Foundation for Statistical Computing.

Raab, D. H. (1962). Statistical Facilitation of Simple Reaction Times. Transactions of the New York Academy of Sciences, 24(5 Series II), 574-590. doi: 10.1111/j.2164-0947 .1962.tb01433.x

Ratcliff, R. (1978). A theory of memory retrieval. Psychological review, 85(2).

Ratcliff, R., Smith, P. L., Brown, S. D., \& McKoon, G. (2016). Diffusion decision model: Current issues and history. Trends in cognitive sciences, 20(4), 260-281.

Rayner, K., Warren, T., Juhasz, B. J., \& Liversedge, S. P. (2004). The Effect of Plausibility on Eye Movements in Reading. Journal of Experimental Psychology: Learning, Memory, and Cognition, 30(6), 1290-1301. doi: 10.1037/0278-7393.30.6.1290

Smith, G. (2018). A theory of timing effects in a self-organizing model of sentence processing (Unpublished doctoral dissertation). University of Connecticut.

Stan Development Team. (2020). RStan: the R interface to Stan. Retrieved from http:// mc-stan.org/ (R package version 2.19.3)

Swets, B., Desmet, T., Clifton, C., \& Ferreira, F. (2008). Underspecification of syntactic ambiguities: Evidence from self-paced reading. Memory \{\&\} Cognition, 36(1), 201216.

Tabor, W., \& Hutchins, S. (2004). Evidence for self-organized sentence processing: diggingin effects. Journal of Experimental Psychology: Learning, Memory, and Cognition, $30(2), 431$.

Traxler, M. J., Pickering, M. J., \& Clifton, C. (1998). Adjunct Attachment Is Not a Form of Lexical Ambiguity Resolution. Journal of Memory and Language, 39(4), 558-592. doi: $10.1006 /$ jmla.1998.2600

Tsay, W.-J., \& Ke, P.-H. (2021). A simple approximation for the bivariate normal integral. Communications in Statistics-Simulation and Computation, 1-14. 
Van Gompel, R., Pickering, M., Pearson, J., \& Liversedge, S. (2005). Evidence against competition during syntactic ambiguity resolution. Journal of Memory and Language, 52(2), 284-307.

Van Gompel, R., Pickering, M. J., \& Traxler, M. J. (2000). Unrestricted race: A new model of syntactic ambiguity resolution. In A. Kennedy, R. Radach, D. Heller, \& J. Pynte (Eds.), Reading as a perceptual process. Oxford: Elsevier.

Van Dyke, J. A., \& McElree, B. (2011). Cue-dependent interference in comprehension. Journal of Memory and Language, 65(3), 247-263. doi: 10.1016/j.jml.2011.05.002

van Gompel, R. P. G., Pickering, M. J., \& Traxler, M. J. (2001). Reanalysis in sentence processing: Evidence against current constraint-based and two-stage models. Journal of Memory and Language, 45(2), 225-258. doi: 10.1006/jmla.2001.2773

Van Zandt, T. (2002). Analysis of response time distributions. Stevens' handbook of experimental psychology, 4, 461-516.

Vasishth, S. (2021, Aug). A common mistake in data analysis (in psychology/linguistics): Subsetting data to carry out nested analyses (part 1 of 2). Retrieved from https://vasishth-statistics.blogspot.com/2021/08/a-common -mistake-in-psychology-and.html

Vasishth, S., Nicenboim, B., Engelmann, F., \& Burchert, F. (2019). Computational models of retrieval processes in sentence processing. Trends in cognitive sciences, 23(11), 968-982.

von der Malsburg, T., \& Vasishth, S. (2013). Scanpaths reveal syntactic underspecification and reanalysis strategies. Language and Cognitive Processes, 28(10), 1545-1578. doi: 10.1080/01690965.2012.728232

Vosse, T., \& Kempen, G. (2009). In Defense of Competition During Syntactic Ambiguity Resolution. Journal of Psycholinguistic Research, 38(1), 1-9. doi: 10.1007/s10936 -008-9075-1

Wickelgren, W. A. (1977). Speed-accuracy tradeoff and information processing dynamics. Acta Psychologica, 41(1), 67-85. doi: 10.1016/0001-6918(77)90012-9

Wickelgren, W. A., Corbett, A. T., \& Dosher, B. A. (1980). Priming and retrieval from short-term memory: A speed accuracy trade-off analysis. Journal of Verbal Learning and Verbal Behavior, 19(4), 387-404. doi: 10.1016/S0022-5371(80)90276-5

Wickens, T. D. (2002). Elementary signal detection theory. Oxford University Press, USA.

Wickham, H., Averick, M., Bryan, J., Chang, W., McGowan, L. D., François, R., .. Yutani, H. (2019). Welcome to the tidyverse. Journal of Open Source Software, 4(43), 1686. doi: $10.21105 /$ joss.01686

Wright, D. B., \& London, K. (2009). Multilevel modelling: Beyond the basic applications. British Journal of Mathematical and Statistical Psychology, 62(2), 439-456.

\section{Appendix}



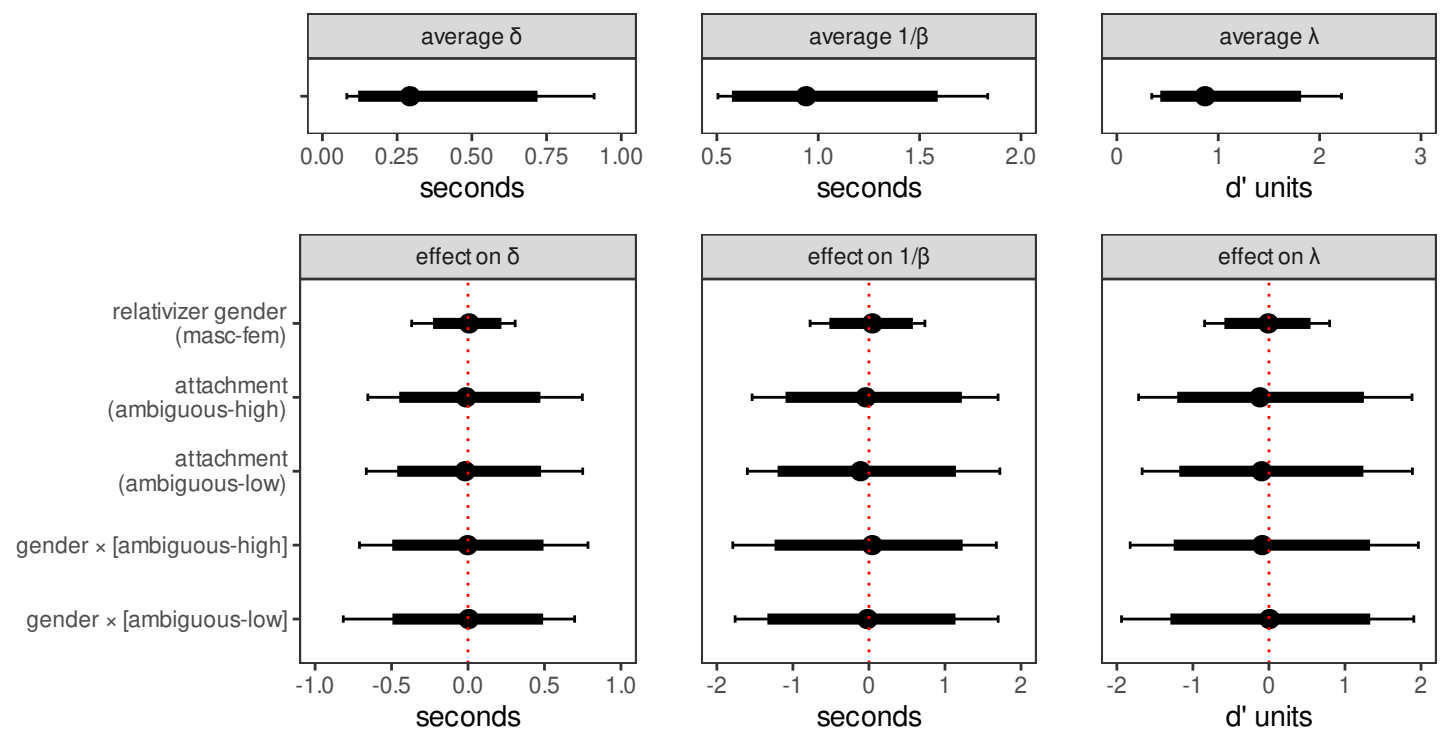

\section{Figure A1}

Credible intervals for the prior distributions for coefficients of a non-linear hierarchical Bayesian model, by speed-accuracy trade-off function parameter (intercept, 1/rate, asymptote). Thin lines represent $90 \% \mathrm{CrIs}$ and thick lines represent $80 \% \mathrm{CrIs}$. Priors for all coefficients involving pairwise differences between the ambiguous condition and an unambiguous conditions are wider than the prior for the effect of gender because they involve two coefficients, as the model was estimated with sum contrasts for attachment. 

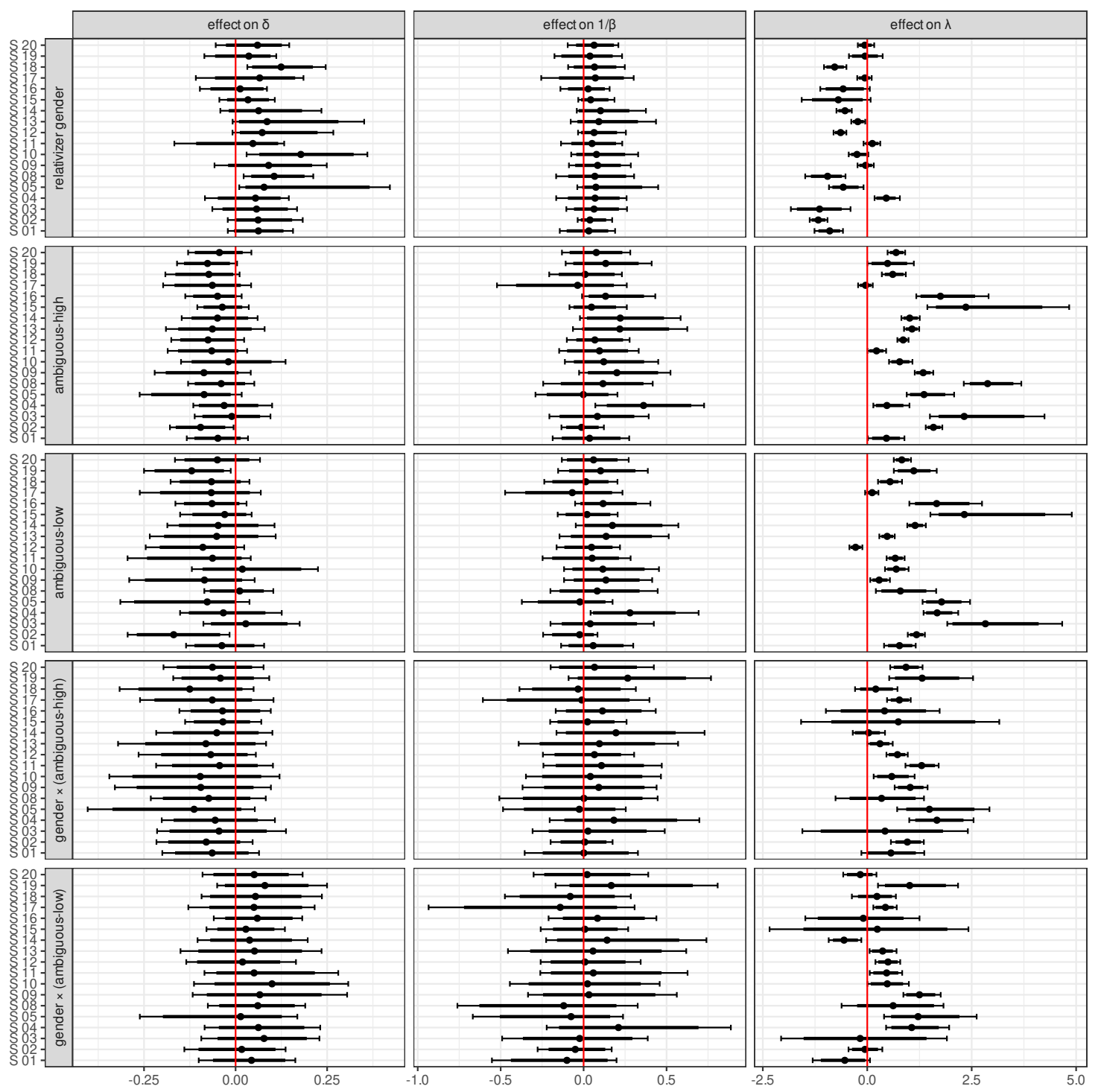

Figure A2

By-subject credible intervals for the coefficients of a non-linear hierarchical Bayesian model, by speed-accuracy trade-off function parameter (intercept, 1/rate, asymptote). Thin lines represent $90 \%$ CrIs and thick lines represent $80 \%$ CrIs. The y-axis represents participant IDs. 

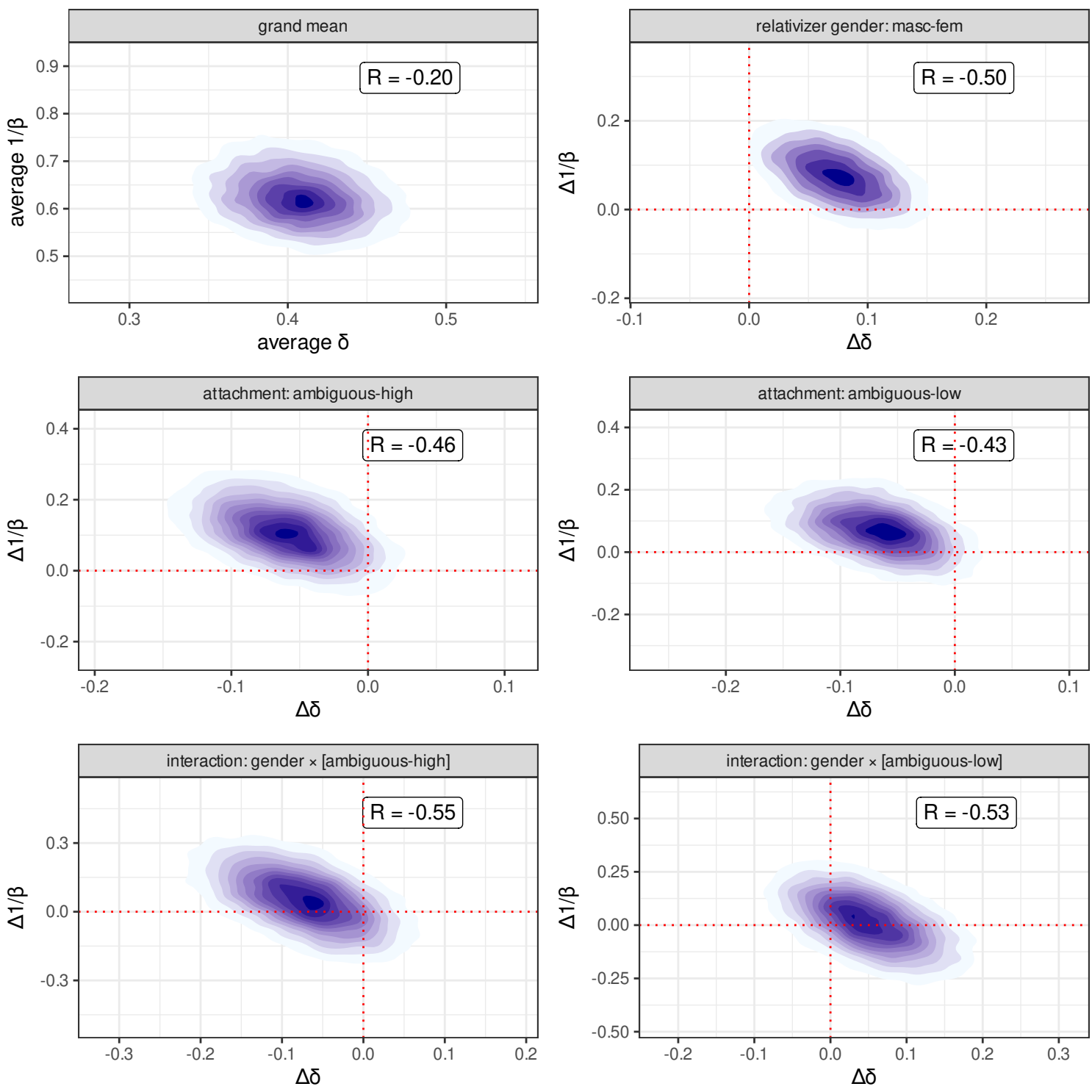

Figure A3

Heatmaps of posterior samples for the coefficients for the SATF intercept ( $\delta, x$-axis), and reciprocal rate $\left(\beta^{-1}, y\right.$-axis). 